\title{
Spectral Theory, Jacobi Matrices, Continued Fractions and Difference Operators
}

\author{
Ahmed Lesfari \\ Chouaïb Doukkali University, Faculty of Sciences, Department of Mathematics, B.P. 20, 24000 El Jadida, Morocco.
}

\author{
Article Info \\ Keywords: Cohomology group, Contin- \\ ued fraction, Deformation, difference \\ operators, Integrable systems, Jacobian \\ varieties, Lax equations, Periodic Ja- \\ cobi matrices, Spectral curves \\ 2010 AMS: $37 \mathrm{~K} 10,32 \mathrm{C} 35,14 \mathrm{~B} 12$, \\ 14H40, 35S05, 11A55, 37K20. \\ Received: 14 March 2019 \\ Accepted: 10 June 2019 \\ Available online: 17 June 2019
}

\begin{abstract}
The aim of this paper is to describe some connections between spectral theory in infinite dimensional Lie algebras, deformation theory and linearization of nonlinear dynamical systems. We explain how results from isospectral deformations, cohomology groups and algebraic geometry can be used to obtain insight into integrable systems. Another part will be dedicated to the study of infinite continued fractions and isospectral deformation of periodic Jacobi matrices and general difference operators from an algebraic geometrical point of view. Also, the notion of algebraically completely integrable systems is explained and techniques to solve such systems are presented. Several nonlinear problems in mathematical physics illustrate these results.
\end{abstract}

\section{Introduction}

The discovery towards the end of the 19th century by Poincaré [1] that complete integrability is an exceptional a phenomenon for Hamiltonian dynamical systems marked the end of a long and fruitful interaction between Hamiltonian mechanics and algebraic geometry and the interest in integrable systems disappeared almost completely; it has been a dormant subject for more than half a century. In fact many algebraic geometrical results such that elliptic and hyperelliptic curves, Abelian integrals, Riemann surfaces, etc., have their origin in problems of mechanics. Fortunately the discovery, 50 years ago that the Korteweg-de Vries (KdV) equation [2] could be integrated by spectral methods have generated an enormous number of new ideas in the area of Hamiltonian completely integrable dynamical systems. The resolution of this problem has led to unexpected connections between mechanics, spectral theory, Lie groups, algebraic geometry and even differential geometry, which has provided new insights into the old mechanical problems of the last centuries and many new ones as well. With respect to this, some questions arise: how do you decide about the complete integrability of a Hamiltonian system? Once you have found necessary conditions of complete integrability on the parameters involved in a Hamiltonian system, how do you prove that the system is effectively completely integrable and how to determine its solutions explicitly? It is well known that solving explicitly a nonlinear Hamiltonian system by quadrature (i.e., by a finite number of algebraic operations including the inverting of functions), was a central theme in mechanics during the 19-th century but the methods of resolution were something very unsystematic and required a great deal of luck and ingenuity. Jacobi [3] himself was very much aware of this difficulty in his famous "Vorlesungen über Dynamik", in the context of geodesic flow on the ellipsoid (before introducing the elliptic coordinates). Difficulties come from the fact that in most problems the quadratures were obtained in terms of elliptic or hyperelliptic integrals and where it was often necessary to find remarkable coordinates algebraically related to the originally given ones, in which the Hamilton-Jacobi equation could be solved by separation of variables. In recent years, important results have been obtained following studies on the Korteweg-de Vries (K-dV) and Kadomtsev-Petviashvili (KP) hierarchies. The use of tau functions related to infinite dimensional Grassmannians, Fay identities, vertex operators and the Hirota's bilinear formalism led to obtaining important results concerning these algebras of infinite order differential operators. In addition, many problems related to algebraic geometry, combinatorics, probabilities and quantum gauge theory,..., have been solved explicitly by methods inspired by techniques from the study of dynamical integrable systems. An account of these results will appear elsewhere. This circle of ideas are far from being completely understood, but it is a gold mine of research problems. 
The purpose of this paper is to describe some connections between spectral theory, Jacobi matrices, continued fractions and difference operators and it is organized as follows: Section 2 concerns nonlinear integrable dynamical systems which can be written as Lax equations with a spectral parameter. Such equations have no a priori Hamiltonian content. However, through the Adler-Kostant-Symes construction, we can produce Hamiltonian dynamical systems on coadjoint orbits in the dual space to a Lie algebra whose equations of motion take the Lax form. We outline an algebraic-geometric interpretation of the flows of these systems, which are shown to describe linear motion on a complex torus via the van Moerbeke-Mumford linearization method. We also present Griffith's method of studying these problems without reference to Kac-Moody's algebras. These results are exemplified by several problems of dynamical integrable systems: Euler-Arnold equations for the geodesic flow on the special orthogonal group (the rotation group), Jacobi geodesic flow on the ellipsoid, Neumann problem on the sphere, Lagrange top, periodic infinite band matrix, $n$-dimensional rigid body and Toda lattice. Section 3 is devoted to the study of some connections between continued fractions, isospectral deformation of Jacobi matrices, difference operators, Cauchy-Stieltjes transform and Abelian integrals from an algebraic geometrical point of view. In Section 4 the notion of algebraically completely integrable Hamiltonian systems are explained and techniques to solve such systems are presented. Some important problems will be studied such that: the periodic 5-particle Kac-van Moerbeke lattice, generalized periodic Toda systems, Ramani-Dorizzi-Grammaticos (RDG) series of integrable potentials and a generalized Hénon-Heiles system.

\section{Coadjoint orbits in Kac-Moody Lie algebras, isospectral deformations and linearization}

Assume a Hamiltonian system having the Lax form (with a rational indeterminate $h$ ) :

$$
\dot{A} \equiv \frac{d A}{d t}=[A, B] \text { or }[B, A], \quad A=\sum_{j=l}^{n} A_{j} h^{j}, \quad B=\sum_{j=l}^{n} B_{j} h^{j},
$$

where $A_{j}$ and $B_{j}$ are matrices.

Theorem 2.1. For every $h \in \mathbb{C}$, the flow (2.1) preserves the spectrum of $A$. For almost all $(z, h) \in \mathbb{C}^{2}$, the spectral curve defined by

$$
C=\left\{(z, h) \in \mathbb{C}^{2}: P(z, h) \equiv \operatorname{det}(A-z I)=0\right\},
$$

is time independent and its coefficients $\operatorname{tr}\left(A^{n}\right)$ are first integrals.

The matrix $A-z I$, has a one-dimensional null-space, defining a holomorphic line bundle on the curve $C$. Whenever the entries of the $A_{j}$ are moving in time, the curve $C$ does not move, inducing a motion on the set of line bundles. The set of holomorphic line bundles on an algebraic curve form a group for the operation of tensoring $\otimes$ and the full set with a given topological type is parametrized by the points of a $g$-dimensional complex algebraic torus, where $g$ is the genus of the curve. This torus that we note, Jac $(C)$, is the Jacobian or Picard variety of the curve. When $C$ is an elliptic curve, $\operatorname{Jac}(C)$ is isomorphic to $C$. Since the flow (2.1) induces deformations of line bundles, their topological type remains unchanged and therefore it induces a motion on the Jacobian variety; under some checkable condition on $A$ and $B$, du to Griffiths [4] (see further for details).

We state the Adler-Kostant-Symes theorem [5]-[7] valid for any Lie algebra :

Theorem 2.2. Let $\mathscr{G}$ be a Lie algebra with a non-degenerate, ad-invariant metric $\langle$,$\rangle . Assume that \mathscr{G}=\mathscr{L} \oplus \mathscr{K}$ as a vector space decomposition, where $\mathscr{L}$ is an ideal and $\mathscr{K}$ is a Lie sub-algebra.

a) Then we have the split $\mathscr{G}=\mathscr{G}^{*}=\mathscr{L}^{\perp}+\mathscr{K}^{\perp}, \mathscr{L}^{\perp} \simeq \mathscr{K}^{*}$ coupled with $\mathscr{K}$ via an induced form $\langle\langle\rangle$,$\rangle inherits the Kostant-Kirillov$ coadjoint symplectic structure. The Poisson bracket of the latter, between functions $F$ and $G$ on $\mathscr{K}^{*}$, is given by

$$
\{F, G\}(A)=\left\langle\left\langle A,\left[\nabla_{\mathscr{K}} F, \nabla_{\mathscr{K}^{*}} G\right]\right\rangle\right\rangle, \quad A \in \mathscr{K}^{*} .
$$

b) Let $M \subset \mathscr{K}^{*}$ be an invariant manifold under the above coadjoint action of $\mathscr{K}$ on $\mathscr{K}^{*}$. Then the functions $H$ defined on a neighborhood of $M$ invariants under the coadjoint action of $\mathscr{G}$, lead to commuting vector fields of the Lax isospectral flows

$$
\dot{A} \equiv \frac{d A}{d t}=\left[A, \operatorname{pr}_{\mathscr{L}}(\nabla H)\right]
$$

where $\operatorname{pr}_{\mathscr{L}}$ is the projection on $\mathscr{L}$.

The reader interested in the most general form of this theorem can consult with profit the recent paper [8]. This is a general theorem for constructing fully dynamical Hamiltonian integrable systems on the coadjoint orbits of a Lie algebra. We will see explicitly how to apply, with some precautions, this theorem to certain Lie algebras of infinite dimension.

Any finite dimensional semi-simple Lie algebra $\mathscr{G}$ leads to an infinite dimensional Lie algebras, the so-called Kac-Moody extensions (that we also note $\mathscr{G})$ :

$$
\mathscr{G}=\left\{\sum_{-\infty}^{n} A_{j} h^{j} \mid n \in \mathbb{Z} \text { free }\right\}
$$

with bracket

$$
\left[\sum A_{i} h^{i}, \sum B_{j} h^{j}\right]=\sum_{k} \sum_{i+j=k}\left[A_{i}, B_{j}\right] h^{k}
$$

and ad-invariant, symmetric forms

$$
\left\langle\sum A_{i} h^{i}, \sum B_{j} h^{j}\right\rangle_{k}=\sum_{i+j=-k}\left\langle A_{i}, B_{j}\right\rangle
$$


depending on $k \in \mathbb{Z}$. Obviously if the form $\langle$,$\rangle is non degenerate, then the form \langle,\rangle_{k}$ is also.

Let $\mathscr{G}_{r}^{s}(r \leq s)$ be the vector subspace of $\mathscr{G}$, corresponding to powers of $h$ between $r$ and $s$. A first interesting class of problems is obtained by taking $\mathscr{G}=\mathscr{G} l(n, \mathbb{R})$ and by putting the form $\langle,\rangle_{1}$ on the Kac-Moody extension. Then we have the decomposition into Lie sub-algebras

$$
\mathscr{G}=\mathscr{G}_{0}^{\infty}+\mathscr{G}_{-\infty}^{-1} \equiv \mathscr{L}+\mathscr{K},
$$

with $\mathscr{L}=\mathscr{L}^{\perp}, \mathscr{K}=\mathscr{K}^{\perp}$ and $\mathscr{L}=\mathscr{K}^{*}$.

Another class is obtained by choosing any semi-simple Lie algebra $\mathscr{G}$. Then the Kac-Moody extension $\mathscr{G}$ equipped with the form $\langle\rangle=,\langle,\rangle_{0}$ has the natural level decomposition

$$
\mathscr{G}=\sum_{i \in \mathbb{Z}} G_{i},\left[G_{i}, G_{j}\right] \subset G_{i+j}, \quad\left[G_{0}, G_{0}\right]=0, \quad G_{i}^{*}=G_{-i} .
$$

Let $A_{+}=\sum_{i \geq 0} G_{i}$ and $A_{-}=\sum_{i<0} G_{i}$. Then the product Lie algebra $\mathscr{G} \times \mathscr{G}$ has the following bracket and pairing

$$
\left[\left(a_{1}, a_{2}\right),\left(\left(b_{1}, b_{2}\right)\right]=\left(\left[a_{1}, b_{1}\right],-\left[a_{2}, b_{2}\right]\right), \quad\left\langle\left(a_{1}, a_{2}\right),\left(b_{1}, b_{2}\right)\right\rangle=\left\langle a_{1}, b_{1}\right\rangle-\left\langle a_{2}, b_{2}\right\rangle .\right.
$$

It admits the decomposition into $\mathscr{L}+\mathscr{K}$ with

$$
\begin{gathered}
\mathscr{L}=\{(a,-a): a \in \mathscr{G}\}, \quad \mathscr{L}^{\perp}=\{(a, a): l \in \mathscr{G}\}, \\
\mathscr{K}=\left\{\left(c_{1}, c_{2}\right): c_{1} \in A_{-}, c_{2} \in A_{+}, \operatorname{Pr}_{0}\left(c_{1}\right)=\operatorname{Pr}\left(c_{2}\right)\right\}, \quad \mathscr{K}^{\perp}=\left\{\left(c_{1}, c_{2}\right): c_{1} \in A_{-}, c_{2} \in A_{+}, P r_{0}\left(c_{2}+c_{1}\right)=0\right\},
\end{gathered}
$$

where $P r_{0}$ denotes projection onto $G_{0}$. Then from the last theorem, the orbits in $\mathscr{K}^{*}=\mathscr{L}^{\perp}$ possesses a lot of commuting Hamiltonian vector fields of Lax form.

We consider the invariant manifold $M_{n}, n \geq 1$, in $\mathscr{L}=\mathscr{K}^{*}$ defined by the set of

$$
A=\sum_{j=1}^{n-1} A_{j} h^{j}+\mu h^{j}, \quad \mu \equiv \operatorname{diag}\left(\mu_{1}, \ldots, \mu_{n}\right) \text { fixed, } \quad \operatorname{diag}\left(A_{n-1}\right)=0
$$

as well as the $\mathscr{K}$-invariant manifolds $M_{-j}^{k}$ defined by

$$
M_{-j}^{k}=\sum_{i=-j}^{k} L_{i} \subseteq \mathscr{G} \simeq \mathscr{L}^{\perp} .
$$

We state the following theorem [9]-[11]:

Theorem 2.3. a) Let $H=\left\langle f\left(A h^{-j}\right), h^{k}\right\rangle_{1}$, be functions defined on the manifold $M_{n}$ where $f$ are differentiable functions. Then, the equations

$$
\dot{A}=\left[A, \operatorname{Pr}_{\mathscr{K}}\left(f^{\prime}\left(A h^{-j}\right) h^{k-j}\right)\right], \quad A=\sum_{i=0}^{n-1} A_{i} h^{i}+\mu h^{n},
$$

determine integrable Hamiltonian systems whose linearization is carried out on the Jacobian of the curve Cof genus $(m-1)(m n-2) / 2$ defined by (2.2). Moreover, especially for $j=n, k=n+1$, the flow

$$
\dot{A}=\left[A, a d_{v} a d_{\mu}^{-1} A_{n-1}+v h\right]
$$

depends on $f$ by the relation $v_{i}=f^{\prime}\left(\mu_{i}\right)$ only.

b) Let $H\left(a_{1}, a_{2}\right)=f\left(a_{1}\right)$, be functions defined on the manifold $M_{-j}^{k}$ where $f$ are differentiable functions. We have

$$
\dot{a}=\left[a,\left(\operatorname{Pr}^{+}-\frac{1}{2} p r_{0}\right) \nabla H(a)\right]
$$

where $\mathrm{Pr}^{+}$is the projection onto $A_{+}$and these Lax equations are linearized on the Jacobian of a curve whose affine equation is given by the characteristic polynomial of elements in $M_{-j}^{k}$, considered as functions of $h$.

Using the van Moerbeke-Mumford approach [11], one can construct an algebraic map from the complex invariant manifolds of linearizable dynamical systems to the Jacobi variety $\operatorname{Jac}(C)$ or one of its sub-manifolds such as Prym varieties, associated with an algebraic curve determined by the spectral curve $C(2.1)$. The equations that linearize the dynamic system are given by

$$
\sum_{j=1}^{g} \int_{s_{j}(0)}^{s_{j}(t)} \omega_{k}=c_{k} t, \quad 1 \leq k \leq g
$$

where $\left(\omega_{1}, \ldots, \omega_{g}\right)$ is a basis in the space of holomorphic differentials on the curve $C$ of genus $g$.

1) As a first example for $M_{1}$ in the above theorem, we consider $A=X+\mu h$, with $X \in \operatorname{so}(n)$. It is deduced that the Hamiltonian flow (2.3), where $\mu_{i}$ and $v_{i}$ can be taken arbitrarily, is the $0^{t h}$-order in $h$

$$
\dot{X}=[X, \Lambda(X)], \quad \Lambda(X)_{i j}=\lambda_{i j} X_{i j}, \quad \lambda_{i j}=\lambda_{j i}, \quad \lambda_{i j}=\frac{v_{i}-v_{j}}{\mu_{i}-\mu_{j}}
$$


and an identity to first order in $h$. This flow expresses the Euler-Arnold equations [12] for the geodesic flow on the group $S O(n)$, for a left invariant diagonal metric $\Lambda$. The algebraic curve

$$
C=\left\{(z, h) \in \mathbb{C}^{2}: \operatorname{det}(X+\mu h-z I)=0\right\},
$$

has an involution

$$
\sigma: C \longrightarrow C, \quad(z, h) \longmapsto(-z,-h),
$$

exchanging sheets of $C$ over $C_{0}=C / \sigma$. In such a situation, this involution extends by linearity to a map (which will again be denoted by $\sigma), \sigma: J a c(C) \longrightarrow \operatorname{Jac}(C)$ and up some points of order two, the Jacobi variety $\operatorname{Jac}(C)$ splits into an even part, i.e., $\operatorname{Jac}(C)$ and an odd part (Prym variety) denoted $\operatorname{Prym}\left(C / C_{0}\right)$ and defined by

$$
\operatorname{Prym}\left(C / C_{0}\right)=\left(H^{0}\left(C, \Omega^{1}\right)^{-}\right)^{*} / H_{1}(C, \mathbb{Z})^{-},
$$

where $\Omega^{1}$ is the sheaf of holomorphic 1 -forms on the curve $C$ and - means the -1 eigenspace for a vector space on which the involution $\sigma$ acts. We have

$$
\operatorname{Jac}(C)=\operatorname{Jac}\left(C_{0}\right) \oplus \operatorname{Prym}\left(C / C_{0}\right) .
$$

The phase space for this problem is an orbit defined in the group $S O(n)$ by $\left[\frac{n}{2}\right]$ orbit invariants. By Theorem 2.3 , the problem linearizes on $\operatorname{Prym}\left(C / C_{0}\right)$ of dimension $\frac{\frac{n(n-1)}{2}-\left[\frac{n}{2}\right]}{2}$.

2) For another example, consider the case of $M_{2}$ in the above theorem with

$$
A=\mu h^{2}-y \otimes y-h x \wedge y, \quad\left(x, y \in \mathbb{R}^{n}\right),
$$

where $(x, y) \in \mathbb{R}^{2 n}$. In this case, equation (2.3) is reduced to the study of

$$
\dot{A}=\left[A, v h+a d_{\nu} a d_{\mu}^{-1}(y \wedge x)\right]
$$

where $v_{i}=f^{\prime}\left(\mu_{i}\right)$. Explicitly, we can rewrite this equation in the form of a nonlinear dynamic system :

$$
\begin{aligned}
\dot{x} & =-\frac{\partial H_{v}}{\partial y}=-v y-\left(a d_{v} a d_{\mu}^{-1}(y \wedge x)\right) x, \\
\dot{y} & =\frac{\partial H_{v}}{\partial x}=-\left(a d_{v} a d_{\mu}^{-1}(y \wedge x)\right) y
\end{aligned}
$$

where

$$
H_{v}=\frac{1}{2} \sum_{i} v_{i}\left(y_{i}^{2}+\sum_{j \neq i} \frac{\left(x_{i} y_{j}-x_{j} y_{i}\right)^{2}}{\mu_{i}-\mu_{j}}\right) .
$$

Note that in the particular case where

$$
f(z)=\log z, \quad v_{i}=\frac{1}{\mu_{i}},
$$

then this problem is reduced to the study of the well known Jacobi geodesic flow on the ellipsoid :

$$
\frac{x_{1}^{2}}{v_{1}^{2}}+\cdots+\frac{x_{n}^{2}}{v_{n}^{2}}=1 \text {. }
$$

Another special case is where $f(z)=\frac{1}{2} z^{2}, v_{i}=\mu_{i}$. Here the problem is reduced to the study of the Neumann movement (under the influence of the force $-\mu x$ ) of a point on the sphere [13]:

$$
S^{n-1}: x_{1}^{2}+\cdots+x_{n}^{2}=1
$$

According to theorem 2.3, the linearization of the problem related to these two cases is carried out on the Jacobi variety $J a c(\mathscr{H})$, where $\mathscr{H}$ is a hyperelliptic curve of genus $n-1$. For an interesting geometric interpretation of these motions and their relationship with confocal quadrics, theorem of Chasles, geodesic, intersection of two quadrics, K-dV equation, etc., see for example [13]-[17].

3) Another example of $M_{2}, n=3$, in the above theorem, concerns the Lagrange spinning top [18]. It expresses a particular case of the rotational motion of a solid body around a fixed point. Here, we have

$$
A=m h+\gamma+l h^{2}
$$

where $m \in \operatorname{so}(3)$ (angular momentum), $\gamma \in \operatorname{so}(3)$ (unit vector in the direction of gravity), $l=(\alpha+\beta) \varepsilon$ with $\varepsilon \in \operatorname{so}(3)$ (coordinates of the center of mass) and where $(\alpha+\beta, \alpha+\beta, 2 \alpha)$ (inertia tensor in diagonalized form). Here, the linearization of the problem takes place on the Jacobi variety of an elliptic curve, i.e., on the curve itself (see [19] and for higher-dimensional generalizations [20]). 
4) As an example of $M_{-j}^{k}$ in theorem 2.3, b) (see [9]-[11]), we consider the periodic infinite band matrix $M$ of period $n$ having $j+h+1$ diagonals; the spectrum of $M$ is defined by the points $(z, h) \in \mathbb{C}^{2}$ such that

$$
M v(h)=z v(h), \quad v(h)=\left(\ldots, h^{-1} v, v, h v, \ldots\right), \quad v \in \mathbb{C}^{n} .
$$

Let $M_{h}$ be the square matrix obtained from $M$ and let $C$ be the curve whose affine equation is $\operatorname{det}\left(M_{h}-z I\right)=0$. Then the set of infinite band matrices with $j+k+1$ diagonals, in higher dimensions many partial results seem to lead to rigidity. In fact, it was shown that a discrete 2-dimensional Laplacian cannot be deformed, given its periodic spectrum; the proof can be summarized by the observation that the Picard variety of most algebraic surfaces are trivial; the proof that the specific spectral surface defined by the 2-dimensional Laplacian has trivial Picard variety is based on the technique of toroidal embedding, which reduces cohomological computations to combinatorial questions. Finally, inspired by the dynamical systems, Mumford [21] has given a beautiful description of hyperelliptic Jacobians of dimension $g$.

Griffiths [4] has given a necessary and sufficient condition on $B$ (easily checkable), for an equation of the type (2.1) to be linearizable on the Jacobi variety $\operatorname{Jac}(C)$ of its spectral curve defined by (2.2) (although, without reference to Kac-Moody Lie algebras). Indeed, suppose that for every $p(z, h)$ belonging to the curve $C$ of affine equation (2.2), with $\operatorname{dim} \operatorname{ker}(A-z I)=1$ (i.e., the corresponding eigenspace of $A$ is one-dimensional) and generated by a vector $v(t, p) \in \mathbb{C}^{n}$. So, we can find a family of holomorphic mappings which send $(z, h) \in C$ to $\operatorname{ker}(A-z I)$ :

$$
\phi_{t}: C \longrightarrow \mathbb{P}^{n}(\mathbb{C}), \quad p \longmapsto \mathbb{C} v(t, p),
$$

called the eigenvector map associated to the equation (2.1). Let

$$
\Psi(t)=\phi_{t}^{*}\left(\mathscr{O}_{\mathbb{P}^{n}(\mathbb{C})}(1)\right) \in \operatorname{Pic}^{s}(C), \quad s=\operatorname{deg} \phi_{t}(C),
$$

where $\operatorname{Pic}^{s}(C) \cong \operatorname{Jac}(C)$ is the Picard variety of the curve $C$ and $\mathscr{O}_{\mathbb{P}^{n}(\mathbb{C})}(1)$ is the hyperplane line bundle in $\mathbb{P}^{n}(\mathbb{C})$. Obviously the degree of $\Psi(t)$ does not vary with $t$ Let $H$ be the hyperplane class in $\mathbb{P}^{n}(\mathbb{C})$. The Poincaré dual of the class $[C]$ of $C$ coincides with the degree of $C$,

$$
\operatorname{deg} \Psi(t)=\int_{C} \phi_{t}^{*} H=\int_{\phi_{t}(C)} H=\operatorname{deg}(C) .
$$

Since $\Psi(t)$ moves in $\operatorname{Pic}^{s}(C)$ when $t$ varies, then by fixing a line bundle $\Psi(0) \in \operatorname{Pic}^{s}(C)$, the line bundle $\Psi(0)^{-1} \otimes \Psi(t)$ moves in the Jacobian variety $\operatorname{Jac}(C)$. We will determine a necessary and sufficient condition of a cohomological nature on $B$ so that the flow

$$
t \longmapsto \Psi(t),
$$

is linearizing on $\operatorname{Jac}(C)$. By applying cohomological techniques of the theory of deformation, we can find necessary and sufficient conditions to linearize the flow (2.4). Indeed, this is because the tangent space for any deformation is in a proper cohomology group, and according to the theory of duality on algebraic curves, the higher cohomology can always be eliminated. Let's see that with a little more detail. Let $X$ be a complex manifold and

$$
\phi: C \longrightarrow X
$$

a non-constant holomorphic map. Let $\theta_{C}, \theta_{X}$ be the respective tangent sheaves and $\phi_{*}$ the differential of $\phi$. The normal sheaf of $C$ in $X$ is defined by the following exact sequence

$$
0 \longrightarrow \theta_{C} \stackrel{\phi_{*}}{\longrightarrow} \phi^{*} \theta_{X} \longrightarrow N_{\phi} \longrightarrow 0
$$

and let $H^{0}\left(C, N_{\phi}\right)$ (that we also note $H^{0}\left(N_{\phi}\right)$ ) be the Kodaira-Spencer tangent space [22] to the moduli space of (2.5). Let $\phi_{t}: C \longrightarrow X$, $\phi_{0}=\phi$, be a deformation of (2.5). In local product coordinates $(z, t)$ on $\bigcup_{t} C_{t}, w=\left(w^{1}, w^{2}, \ldots, w^{n}\right) \in X$, we show that $\phi_{t}$ is given by $(t, \xi) \longmapsto w(t, \xi)$, i.e., the section $\dot{\phi} \in H^{0}\left(N_{\phi}\right)$ and is locally given by

$$
\left.\frac{\partial w(t, \xi)}{\partial t}\right|_{t=0} \text { modulo } \frac{\partial w(0, \xi)}{\partial z} .
$$

The corresponding cohomological sequence of (2.6) is

$$
H^{0}\left(\theta_{C}\right) \longrightarrow H^{0}\left(\phi^{*} \theta_{X}\right) \longrightarrow H^{0}\left(N_{\phi}\right) \stackrel{\bar{\partial}}{\longrightarrow} H^{1}\left(\theta_{C}\right)
$$

Consider the tangent space $H^{1}\left(\theta_{C}\right)$ to the moduli space of the curve $C$ as well as the tangent $\dot{C} \equiv \bar{\partial}(\dot{\phi}) \in H^{1}\left(\theta_{C}\right)$ to the family of curves $\left\{C_{t}\right\}$. Hence, $H^{0}\left(\phi^{*} \theta_{X}\right) / H^{0}\left(\theta_{C}\right) \subset H^{0}\left(N_{\phi}\right)$ is the tangent space to deformations of (2.5) where, according to theorem 2.1 , the curve $C$ is independent of $t$.

Consider now the Euler exact sequence of vector bundles

$$
0 \longrightarrow \mathscr{O}_{\mathbb{P}^{n}(\mathbb{C})} \stackrel{i}{\longrightarrow} \mathbb{C}^{n} \otimes \mathscr{O}_{\mathbb{P}^{n}(\mathbb{C})}(1) \stackrel{p}{\longrightarrow} \mathscr{O}_{\mathbb{P}^{n}(\mathbb{C})} \longrightarrow 0
$$

Therefore, the following sequences $\left(\Psi(0)=\phi^{*} \mathscr{O}_{\mathbb{P}^{n}(\mathbb{C})}(1)\right)$ :

$$
\begin{aligned}
& 0 \\
& \downarrow \\
& \mathscr{O}_{C} \\
& \downarrow v \\
& \mathbb{C}^{n} \otimes \Psi(0) \\
& \downarrow \\
& 0 \quad \longrightarrow \quad \theta_{C} \quad \stackrel{\phi_{*}}{\longrightarrow} \phi^{*} \Theta_{\mathbb{P}^{n}(\mathbb{C})} \quad \longrightarrow \quad N_{\phi} \quad \longrightarrow 0
\end{aligned}
$$


are exact and the cohomology diagram corresponding to these sequences contains the following part :

$$
\begin{aligned}
& H^{0}\left(C, \mathbb{C}^{n} \otimes \Psi(0)\right) \\
& \downarrow \tau
\end{aligned}
$$

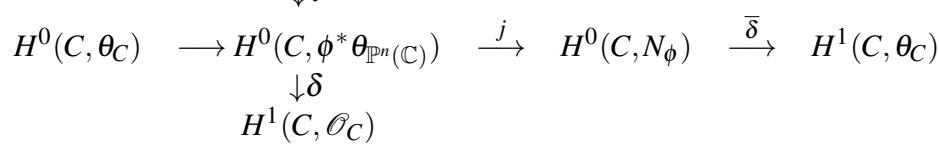

Let $(t, \xi) \longmapsto v(t, \xi) \in \mathbb{C}^{n} \backslash\{0\}$, be a position vector mapping with $\xi$ a local coordinate on $C$. In other words, a local lift $v_{t}$ (which is a time-dependent map $C \longrightarrow \mathbb{C}^{n} \backslash\{0\}$ ) of the family of holomorphic maps $\phi_{t}: C \longrightarrow \mathbb{P}^{n}(\mathbb{C})$, to $\mathbb{C}^{n} \backslash\{0\}$, such that

$$
\phi_{t}(\xi)=\mathbb{C} . v(t, \xi) \subset \mathbb{C}^{n}
$$

As this lift exists only locally, it will have to find an independent object of this lift but which exists globally. The solution is to use this lift to determine such an object that we note $\dot{v}$. Notice that the space $\mathbb{C} v_{t}(p)$ and the fibre of $\phi^{*} \mathscr{O}_{\mathbb{P}^{n}(\mathbb{C})}(-1)$ at a point $p \in C$ identify and define the $\operatorname{maps} \phi^{*} \mathscr{O}_{\mathbb{P}^{n}(\mathbb{C})}(-1) \mathbb{C}^{n} \otimes \mathscr{O}_{C}$ and

$$
v_{t}: \mathscr{O}_{C} \longrightarrow \mathbb{C}^{n} \otimes \Psi(t), \quad \phi \longmapsto \phi v_{t}
$$

(here, $v_{0}$ coincides with the application $v$ mentioned in the previous diagram). In the case where $\eta$ will be another lift such that :

$$
\eta(t, \xi)=\kappa(t, \xi) v(t, \xi), \quad \kappa \neq 0
$$

then we will have $\dot{\eta}=\kappa \dot{v}+\dot{\kappa} v$. The inclusion $\mathscr{O}_{C} \stackrel{v}{\hookrightarrow} \mathbb{C}^{n} \otimes L, L=f^{*} \mathscr{O}_{\mathbb{P}^{n}(\mathbb{C})}(1)$, is locally given by $\mathscr{O}_{\mathscr{C}} \ni \phi \longmapsto \phi \cdot v$, and then (modulo $v(t, \xi))$, the expression

$$
\dot{v}(\xi)=\left.\frac{\partial v(t, \xi)}{\partial t}\right|_{t=0} \in H^{0}\left(C, \mathbb{C}^{n} \otimes L / \mathscr{O}_{C}\right)=H^{0}\left(C, f^{*} \theta_{\mathbb{P}^{n}(\mathbb{C})}\right)
$$

is well-defined independently of the choice of the lift, and we have $\sigma(\dot{v})=\phi$. We are interested in the tangent vector

$$
\left.\dot{\Psi}(0) \equiv \frac{d \Psi(t)}{d t}\right|_{t=0} \in H^{1}\left(C, \mathscr{O}_{C}\right)
$$

Theorem 2.4. If $\dot{v}$ is an infinitesimal variation of $\phi_{t}: C \longrightarrow \mathbb{P}^{n}(\mathbb{C})$, then

$$
\left.\dot{\Psi}(0) \equiv \frac{d \Psi(t)}{d t}\right|_{t=0}=\delta(\dot{v}) \in H^{1}\left(C, \mathscr{O}_{C}\right) .
$$

In addition if $\tau$ is the map mentioned in the diagram above, then there is an equivalence between the fact that $\dot{\Psi}(0)=0$ and $\dot{v}=\tau(w)$ for some $w \in H^{0}\left(\mathbb{C}^{n} \otimes \Psi(0)\right)$.

Let $h_{0}, h_{1}$ be homogeneous coordinates and consider $h$ as an affine coordinate on $\mathbb{P}^{1}(\mathbb{C})$ which is the base of the covering $\pi: C \longrightarrow \mathbb{P}^{1}(\mathbb{C})$. Note that $B(t, h)$ can be written in the form

$$
B(t, h)=\sum_{k=0}^{N} B_{k}(t) h^{k}=\sum_{k=0}^{N} B_{k}(t) h^{N}\left(\frac{h_{1}}{h_{0}}\right)^{k} \in H^{0}(C, \operatorname{Hom}(\mathscr{F}, \mathscr{F}(N))),
$$

where $\mathscr{F}$ is the sheaf of sections of the trivial bundle $C \times \mathscr{F}$. We have $\mathscr{F}(D)=\mathscr{F} \otimes \mathscr{O}_{C}(D)$ and $B(t, h)$ can be seen as a holomorphic section of the bundle $\operatorname{Hom}(\mathscr{F}, \mathscr{F}) \otimes \mathscr{O}_{C}(N), \mathscr{O}_{C}(N)=\pi^{*} \mathscr{O}_{\mathbb{P}^{1}}(N)$. In other words, we visualize $h=\left[h_{0}: h_{1}\right]$ as a homogeneous coordinate on $\mathbb{P}^{1}(\mathbb{C})$ pulled up to $C$. We have $\frac{B}{h_{0}^{K}} \in H^{0}(C, \operatorname{Hom}(\mathscr{F}, \mathscr{F}(D))), v \in H^{0}(V \otimes L)$ where $D=\left(h_{0}^{N}\right)$, is the divisor $N . \pi^{-1}(\infty)$ on the curve $C$ and $\mathscr{F}(D) \cong \mathscr{F}(N)$ are the sections of $\mathscr{F} \otimes \mathscr{O}_{C}(D)$. It should be noted that here $\frac{B}{h_{0}^{N}}$ is a matrix in $\operatorname{Hom}(\mathscr{F}, \mathscr{F})$ with meromorphic functions in $H^{0}\left(C, \mathscr{O}_{C}(D)\right)$ as entries, i.e., $\frac{h_{1}}{h_{0}}$ is seen as a function on $H^{O}\left(C, \mathscr{O}_{C}(D)\right)$. We deduce that $\left(\frac{B}{h_{0}^{N}}\right) \cdot v \in H^{0}(C, \mathscr{F} \otimes \Psi(0)(D))$ and the Lax equation can be interpreted in cohomological form as follows:

Theorem 2.5. The following conditions are equivalent :

(i)

$$
\dot{v}=\tau\left(\frac{B}{h_{0}^{N}} \cdot v\right) \quad \dot{\Psi}(0)=0,
$$

(ii) There is a meromorphic function $\varphi \in H^{O}\left(C, \mathscr{O}_{C}(D)\right)$ such that $\frac{B}{h_{0}^{N}} \cdot v+\varphi v \in H^{0}(C, \mathscr{F} \otimes L(D))$ is holomorphic. 
By differentiating the eigenvalue problem $A v(t, p)=z v(t, p)$ (in the neighborhood of the point $p=(h, z) \in C$ ) with respect to $t$, and taking into account the Lax equation in the form $\dot{A}=[B, A]$, we immediately obtain the expression $A(\dot{v}-B v)=z(\dot{v}-B v)$. Since eigenvalues have (generically) a multiplicity of 1 , then for a some $\lambda$, we have

$$
B v=\dot{v}+\lambda v
$$

This equation can be written in the form $B v=\dot{v}+\lambda_{j} v$, where $\lambda_{j}$ is the main part of Laurent series expansion of $\lambda$ in the neighborhood of $p$. Then given the curve $C$ defined by (2.2) and $p \in C$, Griffiths defines

$$
L_{p} \equiv[\text { Laurent tail }(B)]_{p} \quad=\quad\{\text { main part of Laurent series expansion of } \lambda \text { in the neighborhood of } p\},
$$

and shows that the linearization of the Lax flow takes place on the Jacobi variety $\operatorname{Jac}(C)$ if and only if $p \in(h)_{\infty}(\operatorname{divisor}$ of the poles of $h$ ), we have for any meromorphic function $f$ on $C$ such that : $(f) \geq n(h)_{\infty}$,

$$
\frac{d L_{p}}{d t} \in \text { linear combination }\left\{L_{p} ; \text { Laurent tail at } p \text { of } f\right\}
$$

Let $P(h, g) \in \mathbb{C}[h, g]$ and note that if we replace $B$ by $B+P(h, A)$ in equation (2.1), we see that this equals invariant which shows that $B$ is not unique and that its natural place is somewhere in a cohomology group.

Consider a positive divisor

$$
D=\left(\frac{1}{h}\right)(\infty)=\sum_{j} n_{j} p_{j}, \quad n_{j} \geq 0,
$$

on $C$, where $h$ is seen as a meromorphic function. The polynomial $B(t, h)=\sum_{k=0}^{n} B_{k} h^{k}$ of degree $n$ should be interpreted as an element of $H^{0}\left(C, \operatorname{Hom}(V, V(D))\right.$ where $V$ is the sheaf of sections of the trivial bundle $C \times V$ and $V(D)=V \otimes \mathscr{O}_{C}(D)$. A section of $\mathscr{O}_{D}(D)$ is written

$$
\varphi=\sum \varphi_{j}, \quad \varphi_{j}=\sum_{k=-n_{j}}^{-1} a_{k} z_{j}^{k},
$$

where $z_{j}$ is a local coordinate around $p_{j}$. This is a principal part (Laurent tail) centered on $p_{j}$. A question arises : Given a main part $\varphi_{j}$, determine conditions for a function $\varphi \in H^{0}\left(C, \mathscr{O}_{C}(D)\right)$ such that $\varphi-\varphi_{j}$ is holomorphic in the neighborhood of $p_{j}$. The answer to this question (known as the Mittag-Leffler problem) is provided by

Theorem 2.6. Let $\left\{\varphi_{j}\right\}$ be a Laurent tail and let $D=\sum_{j} a_{j} p_{j}$. The following conditions are equivalent :

(i) There exist $\varphi \in H^{0}\left(C, \mathscr{O}_{C}(D)\right)$ such that $\varphi-\varphi_{j}$ is holomorphic near $p_{j}$.

(ii) For every holomorphic differential $\omega$ on $C$, we have

$$
\sum_{j} \operatorname{Res}_{p_{j}}\left(\varphi_{j} . \omega\right)=0
$$

The residue of $B$, denoted by $\varsigma(B) \in H^{0}\left(C, \mathscr{O}_{D}(\mathscr{D})\right.$, is the collection of Laurent tails $\left\{\lambda_{j}\right\}$ given above, where $\lambda_{j}$ is the main part of the Laurent series expansion of $\lambda$ around $p$.

We will say that the flow $\Psi(t)(2.4)$ is linearized if there is a complex number $c$ such that

$$
\frac{d^{2} \Psi(t)}{d t^{2}}=c \frac{d \Psi(t)}{d t}
$$

The Griffiths theorem is as follows :

Theorem 2.7. 1) We have

$$
\dot{\Psi}(0)=\left.\frac{d \Psi(t)}{d t}\right|_{t=0}=\delta_{1}(\varsigma(B)) .
$$

2) The following conditions are equivalent:

(i) The flow $\Psi(t)$ (2.4) is linearized in $\operatorname{Pic}^{s}(C)$.

(ii) We have

$$
\varsigma(\dot{B})=0 \bmod .(\varsigma(B), \text { Im res }),
$$

where Im res $\subset H^{0}\left(C, \mathscr{O}_{D}(D)\right.$ is the Laurent tails of meromorphic functions in $H^{0}\left(C, \mathscr{O}_{D}(D)\right)$.

(iii) We have

$$
\left.\left.\sum_{j} \operatorname{Res}_{p_{j}}\left(\dot{\zeta}_{j}(B)\right) \omega\right)=t \sum_{j} \operatorname{Res}_{p_{j}}\left(\varsigma_{j}(B)\right) \omega\right), \quad \omega \in H^{0}\left(C, \Omega_{C}\right)
$$


It follows from the above theorem that the linearized flow on $\operatorname{Jac}(C)$ is provided by the bilinear map

$$
\left.(t, \omega) \longmapsto t \sum_{j} \operatorname{Res}_{p_{j}}\left(\varsigma_{j}(B)\right) \omega\right)=t \sum_{j} \operatorname{Res}_{p_{j}}\left(\lambda_{j} \omega\right) .
$$

As an example, consider Euler's problem of a free rigid body in $\mathbb{R}^{n}$. This one is described by the following equations :

$$
\dot{M}=[M, \Omega], \quad M=\Omega J+J \Omega \in \operatorname{so}(n), \quad \Omega(t) \in \operatorname{so}(n),
$$

where $J=\operatorname{diag}\left(\lambda_{1}, \ldots, \lambda_{n}\right), \lambda_{j}>0$. These equations form a Hamiltonian system on each adjoint orbit of $s o(n)$ and whose Hamiltonian is explicitly described by $H(M)=\frac{1}{2}(M, \Omega)=-\frac{1}{4} \operatorname{Tr}(M \Omega)$. Manakov [23] observed that these equations admit a Lax equation with an indeterminate parameter $h$,

$$
\overbrace{\left(M+J^{2} h\right.})=\left[M+J^{2} h, \Omega+J h\right] .
$$

Hence $D=\left(\frac{1}{h}\right)(\infty)=\sum_{j} p_{j}$, is the divisor with $n$ distinct points $p_{j}$ located on $h=\infty$. We deduce from equation (2.7) with $B=\Omega+J h$, the following relation : $\varsigma(B)=\sum_{j} \frac{\lambda_{j}}{z_{j}}$, where $z_{j}=\frac{1}{h}$ is a local coordinate on $C$ around $p_{j}$. We have $\varsigma(\dot{B})=0$ since $\lambda_{i}$ are constant, and consequently, the flow is linearized on $\operatorname{Jac}(C)$. Taking into account that $A=M+J^{2} h, M+M^{\top}=0, J^{2}-J^{2^{\top}}=0$, we obtain $P(h, z)=(-1)^{n} P(-h,-z)$. The curve $C$ has an involution $\sigma: C \longrightarrow C, \quad(h, z) \longmapsto(-h,-z)$. Here the linearization of the problem necessitates the knowledge of $\frac{1}{2} \operatorname{dim} \mathscr{O}$ independent first integrals and in involution (this is because $\Omega$ moves on an adjoint orbit $\mathscr{O} \subset \operatorname{so}(n)$ ). In general, we have

$$
\operatorname{dim} \mathscr{O}=\frac{n(n-1)}{2}-\left[\frac{n}{2}\right]
$$

Let $g(C)=\frac{(n-1)(n-2)}{2}$ be the genus of the algebraic curve $C$ and $g\left(C_{0}\right)$ the genus of the quotient $C_{0}=C / \sigma$ of $C$ by the involution $\sigma$. Using the Riemann-Hurwitz formula, we get

$$
g(C)-g\left(C_{0}\right)=\frac{1}{2}\left(\frac{n(n-1)}{2}-\left[\frac{n}{2}\right]\right)
$$

Note that $\sigma(\varsigma(B))=-\varsigma(B)$ and the linearization of the problem in question is carried out on the Prym variety $\operatorname{Prym}\left(C / C_{0}\right)$ of the curve $C$ for the involution $\sigma$, interchaging the sheets of the double covering $C \longrightarrow C_{0}$. From (2.10) it follows that

$$
\operatorname{dim} \operatorname{Prym}\left(C / C_{0}\right)= \begin{cases}\frac{n(n-2)}{4} & n \equiv 0 \bmod .2 \\ \frac{(n-1)^{2}}{4} & n \equiv 1 \bmod .2\end{cases}
$$

and taking into account (2.9), we finally get $\operatorname{dim} \operatorname{Prym}\left(C / C_{0}\right)=\frac{1}{2} \operatorname{dim} \mathscr{O}$. The linearization of the Euler equations is carried out on the Prym variety $\operatorname{Prym}\left(C / C_{0}\right)$ of exactly the correct dimension.

\section{Infinite continued fraction and spectral theory for periodic Jacobi operators}

A Jacobi matrix is a doubly infinite matrix $\left(a_{i j}\right)$ with entries $i, j$ such that $: a_{i j}=0$ for $|i-j|$ large enough. The set of these matrices is an associative algebra and consequently a Lie algebra by anti-symmetrization. Consider the Jacobi matrix

$$
\Gamma=\left(\begin{array}{ccccc}
b_{1} & a_{1} & 0 & \cdots & 0 \\
a_{1} & b_{2} & a_{2} & & \vdots \\
0 & a_{2} & \ddots & \ddots & 0 \\
\vdots & & \ddots & \ddots & \\
0 & \cdots & 0 & &
\end{array}\right)
$$

where all the $b_{j}$ are real and all the $a_{j}$ are positive, and let

$$
\varphi(z)=\frac{a_{0}^{2}}{z-b_{1}-\frac{a_{1}^{2}}{z-b_{2}-\frac{a_{2}^{2}}{z-b_{3}-} \cdot}}
$$

be the associated continued $\Gamma$-fraction, where $a_{0}$ is a positive real number. By cutting off the $\Gamma$-fraction $\varphi(z)$ at the $k$-th term, we obtain the $k$-th Padé approximant $\frac{A_{k}(z)}{B_{k}(z)}$ of $\varphi(z)$, i.e.,

$$
\varphi(z)=\lim _{k \rightarrow \infty} \frac{A_{k}(z)}{B_{k}(z)}
$$


We show that $\varphi(z)$ admits formal series expansion arount the point $z=0$ (pole),

$$
\varphi(z)=\frac{c_{0}}{z}+\frac{c_{1}}{z^{2}}+\frac{c_{2}}{z^{3}}+\cdots=\sum_{k=0}^{\infty} \frac{c_{k}}{z^{k+1}} .
$$

Note that the characteristic polynomial

$$
B_{k}(z)=\operatorname{det}\left(\begin{array}{ccccc}
b_{1}-z & a_{1} & 0 & \ldots & 0 \\
a_{1} & b_{2}-z & a_{2} & & \vdots \\
0 & a_{2} & \ddots & \ddots & 0 \\
\vdots & & \ddots & \ddots & a_{k-1} \\
0 & \ldots & 0 & a_{k-1} & b_{k}-z
\end{array}\right),
$$

of $\Gamma$, is the last term of the second order recursion

$$
B_{k}(z)=\left(b_{k}-z\right) B_{k-1}(z)-a_{k-1}^{2} B_{k-2}(z) .
$$

The polynomials $A_{k}(z), B_{k}(z)$ form a pair of solutions of a finite difference equation of the second order (the eigenvectors of the Jacobi matrix from which we remove the first row and the first column) :

$$
a_{k+1} y_{k+2}+b_{k+1} y_{k+1}+a_{k} y_{k}=z y_{k+1}, \quad k \in \mathbb{N}
$$

with the boundary conditions : $y_{0} \neq 0, y_{1}=0, y_{N+1}=0$. In addition, these solutions are linearly independent and we have also the following relation :

$$
a_{k-1}\left(A_{k-1}(z) B_{k}(z)-A_{k}(z) B_{k-1}(z)\right)=1, \quad k \in \mathbb{N}^{*}
$$

The polynomials $B_{k}$ form an orthogonal system with respect to the Stieltjes measure $d \sigma(x)$ on $\mathbb{R}$,

$$
\int_{-\infty}^{\infty} B_{k}(x) B_{l}(x) d \sigma(x)=\delta_{k l}
$$

Conversely, if a family of polynomials $P_{n}(x)$ is orthogonal for $d \sigma(x)$, then $P_{n}(x)$ satisfies the following recurrence relation :

$$
P_{k}(x)-\left(\lambda_{k} x-\mu_{j}\right) P_{k-1}(x)+\gamma_{k-1} P_{k-2}(x)=0,
$$

where $\lambda_{k}>0, \mu$ and $\gamma_{k}>0$ are constants. Moreover, if we consider the continued fraction

$$
\psi(z)=\frac{\gamma_{0}}{\lambda_{1} z-\mu_{1}-\frac{\gamma_{1}}{\lambda_{2} z-\mu_{2}-\frac{\gamma_{2}}{\lambda_{3} z-\mu_{3}-} \cdot}}
$$

and realize an equivalent transformation

$$
\psi(z)=\frac{\gamma_{0}}{z-\frac{\mu_{1}}{\lambda_{1}}-\frac{\frac{\gamma_{1}}{\lambda_{1} \lambda_{2}}}{z-\frac{\mu_{2}}{\lambda_{2}}-\frac{\frac{\gamma_{2}}{\lambda_{2} \lambda_{3}}}{z-\frac{\mu_{3}}{\lambda_{3}}-} \cdot}}
$$

we reconstruct the $\Gamma$-fraction corresponding to $d \sigma(x)$ (where we can put $\frac{\gamma_{k}}{\lambda_{k} \lambda_{k+1}}=a_{k}^{2}$ and $\frac{\mu_{k}}{\lambda_{k}}=b_{k}$ ). As a result, there is a one-to-one correspondence between the set of orthogonal polynomial systems on $\mathbb{R}$ and that of Jacobi matrices. In fact, if the orthogonal polynomials

$$
P_{n}=\frac{\gamma_{0}}{\prod_{k=1}^{n-1} a_{k}} B_{n-1}(x), \quad 1 \leq n<\infty
$$

form a basis of the vector space consisting of all the polynomials, then the Jacobi matrix represents the multiplication by $x$.

As an example of $V_{-j, k}$ (theorem 2.3, b)), consider the infinite matrix :

$$
A=\left(\begin{array}{ccccccc}
\ddots & \ddots & & & & & \\
\ddots & b_{0} & a_{0} & 0 & \cdots & 0 & \\
& a_{0} & b_{1} & a_{1} & & \vdots & \\
& 0 & a_{1} & \ddots & \ddots & 0 & \\
\vdots & & \ddots & \ddots & a_{N-1} & \\
& \cdots & 0 & a_{N-1} & b_{N} & \ddots \\
& & & & & \ddots & \ddots
\end{array}\right), \quad\left(a_{i}, b_{i} \in \mathbb{C}\right)
$$


The matrix $A$ is $N$-periodic when

$$
a_{i+N}=a_{i}, \quad b_{i+N}=b_{i},
$$

for all $i \in \mathbb{Z}$. We denote by $f=\left(\ldots, f_{-1}, f_{0}, f_{1}, \ldots\right)$ the (infinite) column vector and by $D$ (shift operator) the operator passage of degree +1 , $D f_{i}=f_{i+1}$. Since the matrix $A$ is $N$-periodic, we have $A D^{N}=D^{N} A$. Reciprocally, this relation of commutation means that $N$ is the period of $A$. Consider the finite Jacobi matrix (symmetric tridiagonal and $N$-periodic) :

$$
A(h)=\left(\begin{array}{ccccc}
b_{1} & a_{1} & 0 & \cdots & a_{N} h^{-1} \\
a_{1} & b_{2} & a_{2} & & \vdots \\
0 & a_{2} & \ddots & \ddots & 0 \\
\vdots & & \ddots & \ddots & a_{N-1} \\
a_{N} h & \cdots & 0 & a_{N-1} & b_{N}
\end{array}\right),
$$

where $h \in \mathbb{C}^{*}$. The determinant of the matrix

$$
A(h)-z I=\left(\begin{array}{ccccc}
b_{1}-z & a_{1} & 0 & \ldots & a_{N} h^{-1} \\
a_{1} & b_{2}-z & a_{2} & & \vdots \\
0 & a_{2} & \ddots & \ddots & 0 \\
\vdots & & \ddots & \ddots & a_{N-1} \\
a_{N} h & \ldots & 0 & a_{N-1} & b_{N}-z
\end{array}\right),
$$

is

$$
\operatorname{det}(A(h)-z I)=(-1)^{N+1}\left(\alpha\left(h+h^{-1}\right)-P_{N}(z)\right) \equiv F\left(h, h^{-1}, z\right)
$$

where $(z, h) \in \mathbb{C} \times \mathbb{C}^{*}, \alpha=a_{1} a_{2} \cdots a_{N}$, and $P(z)$ is given by the following polynomial of degree $N$ (with real coefficients) :

$$
P(z)=\operatorname{det}\left(\begin{array}{ccccc}
b_{1}-z & a_{1} & 0 & \cdots & 0 \\
a_{1} & b_{2}-z & a_{2} & & \vdots \\
0 & a_{2} & \ddots & \ddots & 0 \\
\vdots & & \ddots & \ddots & a_{N-1} \\
0 & \ldots & 0 & a_{N-1} & b_{N}-z
\end{array}\right)-a_{0}^{2} \operatorname{det}\left(\begin{array}{ccccc}
b_{2}-z & a_{2} & 0 & \cdots & 0 \\
a_{2} & b_{3}-z & a_{3} & & \vdots \\
0 & a_{3} & \ddots & \ddots & 0 \\
\vdots & & \ddots & \ddots & a_{N-2} \\
0 & \ldots & 0 & a_{N-2} & b_{N-1}-z
\end{array}\right)=z^{N}+\cdots
$$

Let $C$ be the Riemann surface defined by the set of $(z, h) \in \mathbb{C} \times \mathbb{C}^{*}$ such that : Af $=z f$ and $D^{N} f=h f$. In other words, we have

$$
C=\left\{(z, h) \in \mathbb{C} \times \mathbb{C}^{*}: F\left(h, h^{-1}, z\right)=0\right\} .
$$

Assuming that $\alpha \neq 0$, we derive from (3.5) and (3.6) the following relation :

$$
h=\frac{P(z) \pm \sqrt{P^{2}(z)-4 \alpha^{2}}}{2 \alpha} .
$$

Note that $C$ is a hyperelliptic curve with $2 N$ branch points over the roots of the equation : $P(z)= \pm 2 \alpha$ and two points at infinity $\mathscr{P}$ and $\mathscr{Q}$; the point $\mathscr{P}$ covering the case $z=\infty, h=\infty$ while the point $\mathscr{Q}$ is relative to the case $z=\infty, h=0$. The hyperelliptic involution on the curve $C$ maps $(z, h)$ into $\left(z, h^{-1}\right)$ and $C$ can be singular. Using Riemann-Hurwitz formula, we find $g=N-1$ (= genus of $C$ ). The meromorphic function $h$ has neither zero nor poles except in the neighborhood of $z=\infty$. When $z \nearrow_{\infty}$, we have

$$
h \simeq \frac{P(z)}{\alpha}=\frac{z^{N}}{\alpha}+\cdots,
$$

on the sheet + , which shows that $h$ has a pole of order $N$. Similarly, when $z \nearrow \infty$, we have

$$
h=\frac{P(z)-\sqrt{P^{2}(z)-4 \alpha^{2}}}{2 \alpha}=\frac{2 \alpha}{P(z)+\sqrt{P^{2}(z)-4 \alpha^{2}}} \simeq \alpha z^{-N}+\cdots,
$$

on the sheet -, and therefore $h$ has a zero of order $N$. Let $\mathscr{P}$ be the point covering $\infty$ on the sheets + and $\mathscr{Q}$ the two point covering $\infty$ on the sheets -. Therefore the divisor $(h)$ of the function $h$ on the curve $C$ is

$$
(h)=-N \mathscr{P}+N \mathscr{Q} \text {. }
$$

The curve $C$ has an antiholomorphic involution

$$
\sim: C \longrightarrow C, \quad(z, h) \longmapsto(\bar{z}, 1 / \bar{h}),
$$

i.e., the map $\sim: p \longmapsto \widetilde{p}$ is such that : $\widetilde{P}=\mathscr{Q}$. Since the finite matrix $A(h)$ for $|h|=1$ is self-adjoint, then it admits a real spectrum. Therefore, the fixed points of this involution form a set that we write $C^{\sim}$. The latter is determined by the set of $p \in C$ such that $: \widetilde{p}=p$, or it 
is the set of $(z, h)$ such that : $h=1 / \bar{h}$ and $\bar{z}=z$, or what amounts to the same, is the set of $(z, h)$ such that : $|h|=1$. Let $C_{+}$(repectively $C_{-}$) the set of $p \in C$ such that : $|h|>1$ (repectively $|h|<1$ ). Note that $C_{+}$contains the point $\mathscr{P}$ and $C_{-}$contains the point $\mathscr{Q}$. We have $C \backslash C^{\sim}=C_{+} \cup C_{-}$, which shows that the set $C^{\sim}$ divides $C$ into two distinct regions $C_{+}$and $C_{-}$, and so

$$
C=C_{+} \cup C^{\sim} \cup C_{-} .
$$

In fact, $C^{\sim}$ is homologous to zero because $\mathscr{C}^{\sim}$ can be thought of as the boundary between $C_{+}$and $C_{-}$. Moreover, the involution $\sim$ extends to an involution $*$ on the field of meromorphic functions as follows: $\left.\varphi^{*}(p)=\overline{\varphi(\widetilde{p}}\right)$, and on the differential space as follows : $(\varphi d \psi)^{*}=\varphi^{*} d \psi^{*}$, which shows that : $h^{*}=\frac{1}{h}$ and $z^{*}=z$. The matrices $A$ and $D^{N}$ have an eigenvector $f=\left(\ldots, f_{-1}, f_{0}, f_{1}, \ldots\right)$ in common. Such a condition is parameterized by the Riemann surface $C$ (3.6). In the following, appropriate standardization is used by selecting $f_{0} \equiv 1$, from where $F_{N}=h$. Let us therefore $\bar{f}=\left(f_{1}, f_{2}, \ldots, f_{N-1}\right)^{\top}$. Since $\bar{f}$ satisfies $(A(h)-z I) \bar{f}=0$, then we have

$$
f_{k}=\frac{C_{1, k}}{C_{1, l}} f_{l}=\frac{C_{2, k}}{C_{2, l}} f_{l}=\cdots=\frac{C_{N, k}}{C_{N, l}} f_{l}, \quad 1 \leq k, l \leq N,
$$

where $C_{k, l}$ is the $(k, l)$-cofactor of $(A(h)-z I)$, that is to say,

$$
C_{k, l}=(-1)^{k+l} M_{k, l} .
$$

where $M_{k l}$ is the $(k, l)$ minor of the matrix $(A(h)-z I)$, i.e., the determinant of the $N-1$ submatrix obtained by removing the $k^{t h}-$ line and the $l^{\text {th }}$-column of the matrix $\left.(A(h)-z I)\right)$. In particular, we have

$$
f_{k}=\frac{C_{N, k}}{C_{N, N}} h=\frac{C_{k, k}}{C_{k, N}} h
$$

According to matrix (3.4), we note that

$$
\begin{aligned}
& C_{N, 1}=a_{1} a_{2} \cdots a_{N-1}+(-1)^{N} \frac{a_{N}}{h} P_{N-1}, \\
& C_{1, N}=a_{1} a_{2} \cdots a_{N-1}+(-1)^{N} a_{N} h P_{N-1},
\end{aligned}
$$

where $P_{N-1} \equiv(-z)^{N-2}+\cdots$, and similarly, $C_{N, N}=(-z)^{N-1}+\cdots$. To determine the divisor structure of $f_{k}$, one proceeds as follows : for $f_{1}$, we have

$$
\begin{aligned}
\left(f_{1}\right)_{\infty} & =\left(C_{N, 1}\right)_{\infty}+(h)-\left(C_{N, N}\right)_{\infty} \\
& =-(2 N-2) \mathscr{Q}-N \mathscr{P}+N \mathscr{Q}+(N-1) \mathscr{P}+(N-1) \mathscr{Q} \\
& =\mathscr{Q}-\mathscr{P}
\end{aligned}
$$

and for the other $f_{k}$, we consider first the matrix (3.4) shifted by one, i.e.,

$$
\left(\begin{array}{ccccc}
b_{2}-z & a_{2} & 0 & \cdots & a_{1} h^{-1} \\
a_{2} & b_{3}-z & a_{3} & & \vdots \\
0 & a_{3} & \ddots & \ddots & 0 \\
\vdots & & \ddots & b_{N}-z & a_{N} \\
a_{1} h & \cdots & 0 & a_{N} & b_{1}-z
\end{array}\right)
$$

Hence,

$$
\left(\begin{array}{ccccc}
b_{2}-z & a_{2} & 0 & \cdots & a_{1} h^{-1} \\
a_{2} & b_{3}-z & a_{3} & & \vdots \\
0 & a_{3} & \ddots & \ddots & 0 \\
\vdots & & \ddots & b_{N}-z & a_{N} \\
a_{1} h & \ldots & 0 & a_{N} & b_{1}-z
\end{array}\right)\left(\begin{array}{c}
\frac{f_{2}}{f_{1}} \\
\frac{f_{3}}{f_{1}} \\
\vdots \\
\frac{f_{N}}{f_{1}} \\
h
\end{array}\right)=0
$$

and as above, we have $\left(\frac{f_{2}}{f_{1}}\right)_{\infty}=\mathscr{Q}-\mathscr{P}$, which implies that

$$
\left(f_{2}\right)_{\infty}=\left(\frac{f_{2}}{f_{1}}\right)_{\infty}+\left(f_{1}\right)_{\infty}=2 \mathscr{Q}-2 \mathscr{P},
$$

and in general, we get

$$
\left(f_{k}\right)_{\infty}=k \mathscr{Q}-k \mathscr{P} .
$$

Note that the degree of a minimal positive divisor $D$ on the curve $C$ such that : for all $k \in \mathbb{Z},\left(f_{k}\right)+D \geq-k \mathscr{P}+k \mathscr{Q}$, is given by

$$
\operatorname{deg} D=g=N-1 .
$$


We have

$$
\operatorname{dim} \mathscr{L}(D+k \mathscr{P}-(k+1) \mathscr{Q})=0,
$$

for all $k \in \mathbb{Z}$, i.e., the divisor $D$ is regular. To be convinced of this, it suffices to show that the divisor $D$ is general. It means that $\left(\omega_{1}\left(p_{1}\right), \ldots, \omega_{g}\left(p_{g}\right)\right) \neq(0, \ldots, 0)$ where $\left(\omega_{1}, \ldots, \omega_{g}\right)$ is a normalized base of differential forms on $C$ and $p_{1},, \ldots, p_{g} \in C$, or what is equivalent if $\operatorname{dim} \mathscr{L}(D)=1$ where $\mathscr{L}(D)$ denotes the set of meromorphic functions $f$ such that $:(f)+\mathscr{D} \geq 0$, or what amounts to the same if $\operatorname{dim} \Omega(-D)=0$ where $\Omega(D)$ denotes the set of meromorphic differential forms $\omega$ such that the divisor $(\omega)+D \geq 0$. From the regularity of the divisor $D$ and the Riemann-Roch theorem, we deduce

$$
\begin{aligned}
\operatorname{dim} \mathscr{L}(D+k \mathscr{P}) & =\operatorname{dim} \Omega(-D-k \mathscr{P})+g+k-g+1, \\
& =\operatorname{dim} \Omega(-D-k \mathscr{P})+k+1,
\end{aligned}
$$

for an integer $k>g-2$. Therefore, we have $\operatorname{dim} \mathscr{L}(D+k \mathscr{P})=k+1$, because $\operatorname{dim} \Omega(-D-k \mathscr{P})=0$. Moreover, $\mathscr{L}(D+j \mathscr{P})$ is strictly larger than $\mathscr{L}(D+(j-1) \mathscr{P})$, and therefore by lowering the index $j$ down to 0 , it follows that $\operatorname{dim} \mathscr{L}(D)=1$, which shows that the divisor $D$ is general. Let's show now that $\mathscr{D}$ is regular. It suffices to proceed by induction. We have just shown that $\operatorname{dim} \mathscr{L}(D)=1$. Since $f_{0}=1 \notin \mathscr{L}(D-\mathscr{Q})$, it means that $\mathscr{L}(D-\mathscr{Q}) \varsubsetneqq \mathscr{L}(D)$ and that the function $f_{0}=1$ does not belong to the first space but belongs to the second and then, $\operatorname{dim} \mathscr{L}(D-\mathscr{Q})=0$. Assuming that $\operatorname{dim} \mathscr{L}(D+k \mathscr{P}(k+1) \mathscr{Q})=0$, we obtain (taking into account the Riemann-Roch theorem) immediately

$$
\operatorname{dim} \mathscr{L}(D+(k+1) \mathscr{P}-(k+2) \mathscr{Q}) \leq \operatorname{dim} \mathscr{L}(D+k \mathscr{P}-(k+1) \mathscr{Q})+1=1
$$

which implies equality because $f_{k+1}$ belongs to the first space. In addition, we have

$$
\operatorname{dim} \mathscr{L}(D+(k+1) \mathscr{P}-(k+2) \mathscr{Q})=0,
$$

because $f_{k+1}$ does not belong to the space $\mathscr{L}(D+(k+1) \mathscr{P}-(k+2) \mathscr{Q})$, but belongs to the space $\mathscr{L}(D+(k+1) \mathscr{P}-(k+1) \mathscr{Q})$.

Consider now, the differential of $F$ (3.5) while taking into account that $z$ appears only on the diagonal of the matrix $A(h)-z I$. Therefore, we have

$$
-\sum_{i=1}^{N} C_{i i} d z+h \frac{\partial F}{\partial h} \frac{d h}{h}=0
$$

and either

$$
\omega=\frac{-i C_{N N} d z}{h \frac{\partial F}{\partial h}} .
$$

We have

$$
\begin{aligned}
\omega & =\frac{-i \frac{d h}{h}}{\sum_{i=1}^{N} \frac{C_{i i}}{C_{N N}}}, \\
& =\frac{-i \frac{d h}{h}}{\sum_{i=1}^{N} \frac{C_{i i}}{C_{i N}} \cdot \frac{C_{i N}}{C_{N N}}}, \\
& =\frac{-i \frac{d h}{h}}{\sum_{i=1}^{N} \frac{C_{N i}}{C_{N N}} \cdot \frac{C_{i N}}{C_{N N}}} .
\end{aligned}
$$

Taking into account that $C_{i N}=C_{N i}^{*}, 1 \leq i \leq N$, we obtain

$$
\begin{aligned}
\omega & =\frac{-i \frac{d h}{h}}{\sum_{i=1}^{N} \frac{C_{N i}}{C_{N N}} \cdot\left(\frac{C_{i N}}{C_{N N}}\right)^{*}}, \\
& =\frac{-i \frac{d h}{h}}{\sum_{i=1}^{N} f_{i} f_{i}^{*}}, \\
& = \pm \frac{C_{N N} d z}{\sqrt{P^{2}(z)-4 \alpha^{2}}}
\end{aligned}
$$

From this we deduce that $\omega^{*}=\omega$ and in addition, we have $\omega \geq 0$ on $C^{\sim}$. We also have a relation which shows that the scalar product between $f_{k}$ and $f_{l}$ is

$$
\left\langle f_{k}, f_{l}\right\rangle=\int_{\mathscr{C}^{\sim}} f_{k} \cdot f_{l}^{*} \omega=\left\{\begin{array}{rr}
0 & \text { si } k \neq l \\
>0 & \text { si } k=l
\end{array}\right.
$$

That is, the functions $f_{k}, k \in \mathbb{Z}$, are orthogonal to $\mathscr{C}^{\sim}$ with respect to $\omega$. We deduce from these properties that the divisor of $\omega$ is $(\omega)=D+\widetilde{D}-\mathscr{P}-\mathscr{Q}$, for the involution $\sim$ introduced previously. Given a matrix of the form $A(3.3)$, we have obtained a series of data $\{C, z, h, D, \omega\}$. What is remarkable is that the reverse is also true (for further information, see [11]) : 
Theorem 3.1. Consider the following two sets of data :

1) Let $a_{i}, b_{i} \in \mathbb{C}, a_{i} \neq 0$, where $a_{i+N}=a_{i}, b_{i+N}=b_{i},-\infty<i<+\infty$. An infinite $N$-periodic matrix

$$
\left(\begin{array}{ccccccc}
\ddots & \ddots & & & & & \\
\ddots & b_{0} & a_{0} & 0 & \ldots & 0 & \\
& \bar{a}_{0} & b_{1} & a_{1} & & \vdots & \\
& 0 & \bar{a}_{1} & \ddots & \ddots & 0 & \\
& \vdots & & \ddots & \ddots & a_{N-1} & \\
& 0 & \cdots & 0 & \bar{a}_{N-1} & b_{N} & \ddots \\
& & & & & \ddots & \ddots
\end{array}\right),
$$

modulo conjugation by $N$-periodic diagonal matrices with real entries.

2) Let $\mathscr{P}$ and $\mathscr{Q}$ two points on a curve of genus $N-1$ and $D$ be a divisor of degree $N-1$ on $C$ such that:

$$
(h)=-N \mathscr{P}+N \mathscr{Q}, \quad(z)=-\mathscr{P}-\mathscr{Q}+S,
$$

where $h, z$ are two meromorphic functions on $C$ and $S$ is a positive divisor not containing the points $\mathscr{P}$ and $\mathscr{Q}$. The curve $C$ is equipped with an antiholomorphic involution $\sim:(z, h) \longmapsto(\bar{z}, \overline{\bar{h}})$, for which $C=\mathscr{C}_{+} \cup C^{\sim} \cup C_{-}$, where $\mathscr{C}^{\sim}$ is the set of $p \in C$ such that $: \widetilde{p}=p$, i.e., the set of $(z, h)$ such that $:|h|=1$, and $C_{+}$(repectively $C_{-}$) is the set of $p \in C$ such that $:|h|>1$ (repectively $|h|<1$ ) containing $\mathscr{P}$ (repectively $\mathscr{Q}$ ). By introducing an involution $\star$ acting on the space of all meromorphic functions on C and on the differential space in a way $\varphi^{\star}(p)=\widetilde{\varphi(\widetilde{p})}$ and $(\varphi d \psi)^{\star}=\varphi^{\star} d \psi^{\star}$, then $h^{\star}=h^{-1}$ and $z^{\star}=z$ and the divisor of a differential form $\omega$ on $C$ is

$$
(\omega)=D+\widetilde{D}-\mathscr{P}-\mathscr{Q}
$$

Then, there is a one-to-one correspondence and equivalence of these sets of data.

For any difference operator $X$, we define

$$
\left(X_{+}\right)_{i j}=\left\{\begin{array}{rl}
X_{i j} & \text { si } i<j, \\
\frac{1}{2} X_{i j} & \text { si } i=j, \\
0 & \text { si } i>j,
\end{array} \quad X_{-}=X-X^{[+]} .\right.
$$

Let $\mathscr{M}$ be the vector space of infinite $N$-periodic matrices $A$ such that for some $k, a_{i j}=0$ if $|i-j|>K$. On $\mathscr{M}$, we introduce the following scalar product :

$$
\langle A, B\rangle=\operatorname{Tr}\left(A B^{\top}\right)=\sum_{(i, j) \in \mathbb{Z}^{2}} a_{i j} b_{i j}
$$

We call a functional $F$ differentiable if there exists a matrix $\frac{\partial F}{\partial A}$ in $\mathscr{M}$ such that :

$$
\lim _{\varepsilon \rightarrow 0} \frac{F(A+\varepsilon B)-F(A)}{\varepsilon}=\left\langle\frac{\partial F}{\partial A}, B\right\rangle,
$$

for every $B$. The following bracket

$$
\{F, G\}=\left\langle\left[\left(\frac{\partial F}{\partial X}\right)_{+},\left(\frac{\partial G}{\partial X}\right)_{+}\right]-\left[\left(\frac{\partial F}{\partial X}\right)_{-},\left(\frac{\partial G}{\partial X}\right)_{-}\right], X\right\rangle, \quad(F, G \in \mathscr{M}),
$$

satisfies the Jacobi identity. Let $P\left(A, S, S^{-1}\right)$ be a polynomial in $S+S^{-1}$ and $A$ with real coefficients. Consider the following Lax equation:

$$
\dot{A}=\left[P\left(A, S, S^{-1}\right)_{+}-P\left(A, S, S^{-1}\right)_{-}, A\right] .
$$

When the matrix $A(t)$ deforms with $t$, then only the divisor $D$ varies while $\{C, z, h, \mathscr{P}, \mathscr{Q}\}$ remain fixed. As we have already shown, the coefficients of $z^{i} h^{j}$ in equation (3.5) are invariants of this motion. The divisor $D(t)$ evolves linearly on the Jacobi variety Jac $(C)$. Any linear flow over $\operatorname{Jac}(C)$ is equivalent to equation (3.8) and can be written in the form of a Hamitonian vector field with respect to the above bracket. For example, the flow

$$
\dot{A}=\left[A,\left(S^{-k} A^{l}\right]_{+}\right]
$$

is written as follows :

$$
\dot{a}_{i j}=\left\{F, a_{i j}\right\}, \quad F=\frac{1}{l+1} \operatorname{Tr}\left(S^{-k} A^{l+1}\right) .
$$


The (Poisson) bracket of two functional of the form $\operatorname{Tr}\left(S^{-k} A^{l+1}\right)$ is zero, which means that we have a set of integrals in involution. Let $\left(\omega_{1}, \ldots, \omega_{g}\right)$ be a holomorphic differential basis on the hyperelliptic curve $C$. We have

$$
\omega_{k}=\frac{z^{k-1}}{\sqrt{P^{2}(z)-4 Q^{2}}}, \quad k=1,2, \ldots, g
$$

and let $c_{k}=\operatorname{Res}_{p}\left(\omega_{k} z^{j}\right), 1 \leq j \leq g$. Since the order of the zeros of $\omega_{k}$ at the points at infinity $\mathscr{P}, \mathscr{Q}$ is equal to $g-k$, then $c_{k}=0$ for $k=1,2, \ldots, g-j+1$ and $c_{k} \neq 0$ for $k=g-j+1$. Therefore, the flow which leaves invariant the spectrum of $A$ and $X$ is given by a polynomial $P(z)$ of degree at most equal to $g$ :

$$
\dot{A}=\frac{1}{2}\left[A, P(A)_{+}-P(A)_{-}\right],
$$

where $P(A)_{+}$is the upper triangular part of $P(A)$ and $-P(A)_{-}$is the lower triangular part of $P(A)$, including the diagonal of $P(A)$. The (Poisson) bracket between two functional $F$ and $G$ can still be written in the form

$$
\{F, G\}=\left\langle\left(\begin{array}{ll}
\frac{\partial F}{\partial a} & \frac{\partial F}{\partial b}
\end{array}\right), J\left(\begin{array}{l}
\frac{\partial G}{\partial a} \\
\frac{\partial G}{\partial b}
\end{array}\right)\right\rangle,
$$

where $\frac{\partial F}{\partial a}=\left(\frac{\partial F}{\partial a_{i}}\right)$ and $\frac{\partial F}{\partial b}=\left(\frac{\partial F}{\partial b_{i}}\right)$ are the column vectors, while $J$ is the following $2 n$-order antisymmetric matrix :

$$
J=\left(\begin{array}{cc}
O & \mathscr{A} \\
-\mathscr{A}^{\top} & O
\end{array}\right), \quad \mathscr{A}=2\left(\begin{array}{ccccc}
a_{1} & 0 & 0 & \ldots & -a_{N} \\
-a_{1} & a_{2} & 0 & & \vdots \\
0 & -a_{2} & a_{3} & & \vdots \\
\vdots & & & & \vdots \\
0 & \ldots & \ldots & -a_{N-1} & a_{N}
\end{array}\right) .
$$

The symplectic structure [24] is given by

$$
\omega=\sum_{j=2}^{N} d b_{j} \wedge \sum_{j \leq i \leq N} \frac{d a_{i}}{a_{i}} .
$$

Flaschka variables [25] :

$$
a_{j}=\frac{1}{2} e^{x_{j}-x_{j+1}}, \quad b_{j}=-\frac{1}{2} y_{j}
$$

applied to the form (3.9) with $x_{N+1}=0$, leads to the symplectic structure

$$
\omega=\frac{1}{2} \sum_{j=2}^{N} d x_{j} \wedge d y_{j}
$$

used by Moser [26, 27] in the study of a dynamic system describing the motion of $N-1$ particles on a line, interacting under an exponential potential. See also the example below concerning the study of Toda lattice. We have

$$
\left.\operatorname{det}\left(A_{h}-z I\right)\right|_{h=i}=(-1)^{N} z^{N}+\beta_{1} z^{N-1}+\beta_{2} z^{N-2}+\cdots+\beta_{N},
$$

where $\beta_{2}, \ldots, \beta_{N}$ are the $g$ invariant, functionally independent and in involution. These are given by the branch points on the hyperelliptic curve $\mathscr{C}$ or by the quantities $\operatorname{Tr} A^{k}$ for $k=2,3, \ldots, N$, i.e., by the $g=N-1$ points chosen from the spectrum of $A_{1}$ and $A_{-1}$. With Jacobi's matrix, we can associate an operator $T$ on a separable Hilbert space $E$ as follows,

$$
T e_{0}=b_{0} e_{0}+a_{0} e_{1}, \quad T e_{i}=b_{i} e_{j}+a_{i-1} e_{i-1}+a_{i} e_{i+1}, \quad i=1,2, \ldots
$$

where $\left(e_{1}, e_{2}, \ldots\right)$ is an orthonormal basis in $E$. The operator $T$ is symmetric. Indeed, we have $\left\langle T u_{1}, u_{2}\right\rangle=\left\langle u_{1}, T u_{2}\right\rangle$ for any two finite vectors, according to the symmetry of the Jacobi matrix. Moreover, if the Carleman's condition :

$$
\frac{1}{a_{0}}+\frac{1}{a_{1}}+\cdots+\frac{1}{a_{n}}+\cdots=+\infty
$$

is satisfied, then the spectrum of the self-adjoint operator $T$ (with $e_{0}$ a generating element) is simple. In this case, the information about the spectrum of $T$ is contained in the following function,

$$
\varphi(z)=\left\langle(T-z I)^{-1} e_{0}, e_{0}\right\rangle=\int_{-\infty}^{\infty} \frac{d \sigma(x)}{z-x},
$$

defined at $z \notin \sigma(T)$ where $\sigma(x)=\left\langle I_{x} e_{0}, e_{0}\right\rangle$ and $I_{x}$ is the resolution of the identity operator $T$. Recall that the infinite continued fraction converges if the limit (3.2) exists. If the operator $T$ is self-adjoint, then the continued fraction $\varphi(z)$ converges uniformly in any closed bounded domain of $z$ without common points with real axis, to the analytic function defined by (3.10). If the support of $d \sigma(x)$ is bounded, 
then the sequence $\left(\frac{A_{k}(z)}{B_{k}(z)}\right)$ converges uniformly to a holomorphic function near $z=\infty$. Moreover, if a Jacobi matrix is bounded, i.e., if there exists $\rho>0$ such that, for all $j,\left|a_{j}\right| \leq \frac{\rho}{3},\left|b_{j}\right| \leq \frac{\rho}{3}$, then the associated $\Gamma$-fraction converges uniformly on the domain $\{z:|z| \geq \rho\}$ and the support of $d \sigma(x)$ is included in $[-\rho, \rho]$. The fraction $\Gamma$ associated with a periodic Jacobi matrix (this case is obviously bounded ) converges near $z=\infty$. In addition, the function $\varphi(z)$ is written in the form (3.10) (Cauchy-Stieltjes transform of $d \sigma(x)$ ), which shows that $\varphi(z)$ has a first-order zero at $z=\infty$ and for any point $z$ belonging the upper-half plane, the imaginary part of $\varphi(z)$ is non positive.

We will now extend the Jacobi matrix $\Gamma$ to the infinite symmetric, tridiagonal and $N$-periodic Jacobi matrix $A$ (3.3) and use the results obtained previously. We consider $\varphi(z)(3.1)$ as being the associated $N$-periodic $\Gamma$-fraction. The latter converges near the infinite point $z=\infty$. An analytic extension of the function $\varphi(z)$ allows us to see that this coincides with the meromorphic function $a_{0} f_{1}$ on the genus $(N-1)$-hyperelliptic curve $C$ (3.6). This curve is branched at the $2 N$ real zeroes $\xi_{1}, \xi_{2}, \ldots, \xi_{2 N}$ of the polynomial $P^{2}(z)-4 \alpha^{2}$. We define the stable band as being the interval $\left[\xi_{2 j-1}, \xi_{2 j}\right], 1 \leq j \leq N$, and the unstable band the interval $\left[\xi_{2 j}, \xi_{2 j+1}\right], 1 \leq j \leq N-1$.

Theorem 3.2. Each zero $\sigma_{1}<\sigma_{2}<\cdots<\sigma_{N-1}$ of $C_{k, l}$ (3.7), belongs to the $j$-th finite unstable band $\left[\lambda_{2 j}, \lambda_{2 j+1}\right], 1 \leq j \leq N-1$.

We will see below (theorem 3.3) how to express the function $\varphi(z)$ in terms of Abelian integrals on the hyperelliptic curve $C$ (3.6). Note that for $N=1, B_{k}(x)$ is the well-known Chebyshev polynomial of the second kind. In addition, Kato [28, 29] discovered, for $N>1$, new results related to discrete measurements. We have seen that

$$
\varphi(z)=a_{0} f_{1}=a_{0} \frac{C_{N, 1}}{C_{N, N}} h,
$$

belonging to $\mathscr{L}(D+\mathscr{P}-\mathscr{Q})$. Then, we have

Theorem 3.3. We have

$$
\varphi(z)=\frac{\operatorname{Res}_{\sigma_{1}^{-}} \varphi(z)}{z-\sigma_{1}}+\cdots+\frac{\operatorname{Res}_{\sigma_{N-1}^{-}} \varphi(z)}{z-\sigma_{N-1}}+\frac{(-1)^{N+1}}{2 \pi i}\left(\int_{\xi_{1}}^{\xi_{2}} \frac{\sqrt{P^{2}(x)-4 \alpha^{2}}}{(z-x) C_{N, N}(x)} d x+\cdots+\int_{\xi_{2 N-1}}^{\xi_{2 N}} \frac{\sqrt{P^{2}(x)-4 \alpha^{2}}}{(z-x) C_{N, N}(x)} d x\right),
$$

where,

$$
\operatorname{Res}_{\sigma_{j}^{-}} \varphi(z) \equiv \frac{\alpha h\left(\sigma_{j}^{-}\right)+(-1)^{N} a_{0}^{2} \cdot \Delta}{\prod_{l \neq j}\left(\sigma_{j}-\sigma_{l}\right)}, \quad j=1,2, \ldots, N-1
$$

and

$$
\Delta \equiv \operatorname{det}\left(\begin{array}{ccccc}
b_{2}-\sigma_{j} & a_{2} & 0 & \cdots & 0 \\
a_{2} & b_{3}-\sigma_{j} & a_{3} & & \vdots \\
0 & a_{3} & \ddots & \ddots & 0 \\
\vdots & & \ddots & \ddots & a_{N-2} \\
0 & \ldots & 0 & a_{N-2} & b_{N-1}-\sigma_{j}
\end{array}\right) .
$$

The differentials obtained in the previous section,

$$
a \frac{C_{N, N}(x)}{\sqrt{P^{2}(x)-4 \alpha^{2}}} d x, \quad b \frac{\sqrt{P^{2}(x)-4 \alpha^{2}}}{C_{N, N}(x)} d x,
$$

( $a$ and $b$ are constants) are positive mesures on each stable band $\left[\xi_{2 j-1}, \xi_{2 j}\right]$. Therefore, the expression (3.11) means that $\varphi(z)$ can be obtained by the Cauchy-Stieltjes transform of

$$
\left.d \sigma=\sum_{j=1}^{N-1} \operatorname{Res}_{\sigma_{j}^{-}} \varphi(z), \sigma_{j}^{-}\right) \cdot C\left(x-\sigma_{j}\right) d x+\frac{(-1)^{N+1}}{2 \pi i} \cdot \frac{\sqrt{P^{2}(x)-4 \alpha^{2}}}{C_{N, N}(x)} d x=\text { discrete mesure }+ \text { continuous mesure, }
$$

as follows,

$$
\varphi(z)=\int_{-\infty}^{\infty} \frac{d \sigma}{z-x}
$$

The function $\varphi(z)$ belongs to $\mathscr{L}\left(D^{\prime}+\mathscr{P}-\mathscr{Q}\right)$ where $D^{\prime}=\sigma_{1}^{+}+\cdots+\sigma_{N-1}^{+}$is contained in $C_{+}=\{p \in C:|h|>1\}$ (see previous section). From expression (3.11), we have

$$
D=\sigma_{j_{1}}^{-}+\cdots+\sigma_{j_{l}}^{-}+\sigma_{j_{l+1}}^{+}+\cdots+\sigma_{j_{N-1}}^{+},
$$

where $j_{1}<j_{2}<\ldots<j_{l}$ denote the numbers for which $\operatorname{Res}_{\sigma_{j}^{-}} \varphi(z)>0$ and $j_{l+1}<j_{l+2}<\ldots<j_{N-1}$ the numbers for which $\operatorname{Res}_{\sigma_{j}^{-}} \varphi(z)=0$. Hence,

$$
\operatorname{Res}_{\sigma_{j}^{-}} \varphi(z)=0 \text { or }-\frac{\sqrt{P^{2}\left(\sigma_{j}^{-}\right)-4 \alpha^{2}}}{\prod_{l \neq j}\left(\sigma_{j}-\sigma_{l}\right)}
$$


The Toda lattice equations [30] describe the motion of $n$ masses with exponential restoring forces :

$$
H=\frac{1}{2} \sum_{j=1}^{N} y_{j}^{2}+\sum_{j=1}^{N} e^{x_{j}-x_{j+1}}, \text { (Hamiltonian). }
$$

We noted above that Flaschka variables [25] : $a_{j}=\frac{1}{2} e^{x_{j}-x_{j+1}}, b_{j}=-\frac{1}{2} y_{j}$, can be used to express the symplectic structure $\omega$ (3.9) in terms of $x_{j}$ and $y_{j}$,

$$
d a_{j}=a_{j}\left(d x_{j}-d x_{j+1}\right), \quad 2 d b_{j}=-d y_{j}
$$

then

$$
\omega=\frac{1}{2} \sum_{j=2}^{N} d x_{j}^{*} \wedge d y_{j}^{*}, \quad\left(x_{j}^{*} \equiv x_{j}-x_{1}, \quad y_{j}^{*} \equiv y_{j}\right) .
$$

We will study the integrability of this problem with the Griffiths approach. There are two cases :

(i) The non-periodic case, i.e., $x_{0}=-\infty, x_{N+1}=+\infty$, where the masses are arranged on a line. In term of the Flaschka variables above, Toda's equations take the following form

$$
\begin{aligned}
& \dot{a}_{j}=a_{j}\left(b_{j+1}-b_{j}\right), \\
& \dot{b}_{j}=2\left(a_{j}^{2}-a_{j+1}^{2}\right),
\end{aligned}
$$

with $a_{N+1}=a_{1}$ and $b_{N+1}=b_{1}$. To show that this system is completely integrable, one should find $N$ independent first integrals in involution. From the second equation, we have

$$
\overbrace{\left(b_{1}+b_{2}+\cdots+b_{N}\right)}^{i}=\dot{b}_{1}+\dot{b}_{2}+\cdots+\dot{b}_{N}=0
$$

and we normalize $b_{i}$ 's by requiring that $b_{1}+b_{2}+\cdots+b_{N}=0$. Applying this fact to (3.9), leads to the following symplectic form :

$$
\omega=\frac{1}{2} \sum_{j=2}^{N} d x_{j} \wedge d y_{j}
$$

We have obtained a first integral of the system and it will be necessary to determine $N-1$ other integrals that are functionally independent and in involution. We further define $N \times N$ matrices $A$ and $B$ with

$$
A=\left(\begin{array}{ccccc}
b_{1} & a_{1} & 0 & \cdots & a_{N} \\
a_{1} & b_{2} & \vdots & & \vdots \\
0 & \ddots & \ddots & \ddots & 0 \\
\vdots & & \ddots & b_{N-1} & a_{N-1} \\
a_{N} & \cdots & 0 & a_{N-1} & b_{N}
\end{array}\right), \quad B=\left(\begin{array}{ccccc}
0 & a_{1} & \cdots & \cdots & -a_{N} \\
-a_{1} & 0 & \vdots & & \vdots \\
\vdots & \ddots & \ddots & \ddots & \vdots \\
\vdots & & \ddots & \ddots & a_{N-1} \\
a_{N} & \cdots & \cdots & -a_{N-1} & 0
\end{array}\right)
$$

The system in question is written in the form $\dot{A}=[B, A]$. Since $I_{k}=\frac{1}{k} t r A^{k}, k=1,2, \ldots, N$, are first integrals (see theorem 2.1), then

$$
\dot{I}_{k}=\operatorname{tr}\left(\dot{A} \cdot A^{k-1}\right)=\operatorname{tr}\left([B, A] \cdot A^{k-1}\right)=\operatorname{tr}\left(B A^{k}-A B A^{k-1}\right)=0 .
$$

Notice that $I_{1}$ is the first integral already know. These $N$ first integrals are functionally independent and in involution, the system in question is thus completely integrable.

(ii) The periodic case, i.e., $y_{j+N}=y_{j}, x_{j+N}=x_{j}$, the connected masses will be arranged on a circle. We show that in this case, the spectrum of the periodic matrix

$$
A(h)=\left(\begin{array}{ccccc}
b_{1} & a_{1} & 0 & \cdots & a_{N} h^{-1} \\
a_{1} & b_{2} & \vdots & & \vdots \\
0 & \ddots & \ddots & \ddots & 0 \\
\vdots & & \ddots & b_{N-1} & a_{N-1} \\
a_{N} h & \cdots & 0 & a_{N-1} & b_{N}
\end{array}\right)
$$

remains invariant in time. The matrix $B(h)$ depending on the spectral parameter $h$, has the form

$$
B(h)=\left(\begin{array}{ccccc}
0 & a_{1} & \cdots & \cdots & -a_{N} h^{-1} \\
-a_{1} & 0 & \vdots & & \vdots \\
\vdots & \ddots & \ddots & \ddots & \vdots \\
\vdots & & \ddots & \ddots & a_{N-1} \\
a_{N} h & \cdots & \cdots & -a_{N-1} & 0
\end{array}\right)
$$


and the rest follows from the general theory. Note that if $a_{j}(0) \neq 0$, then $a_{j}(t) \neq 0$ for all $t$. Since $A^{\top}(h)=A\left(h^{-1}\right)$, the spectral curve $C$ is given by

$$
0=\operatorname{det}(A(h)-z I)=P\left(\frac{1}{h}, z\right) \equiv P(h, z) .
$$

By the antisymmetry of $A$, the curve $C$ has an involution

$$
\tau: C \longrightarrow C, \quad(h, z) \longmapsto\left(\frac{1}{h}, z\right)
$$

We choose

$$
A(h)=\left(\begin{array}{ccc}
0 & \ldots & a_{N} \\
\vdots & \ddots & \vdots \\
0 & \ldots & 0
\end{array}\right) h^{-1}+\left(\begin{array}{ccccc}
b_{1} & a_{1} & & & \\
a_{1} & b_{2} & & & \\
& & \ddots & & \\
& & & b_{N-1} & a_{N-1} \\
& & & b_{N} & a_{N}
\end{array}\right)+\left(\begin{array}{ccc}
0 & \ldots & 0 \\
\vdots & \ddots & \vdots \\
a_{N} & \ldots & 0
\end{array}\right) h .
$$

Note that here the matrix $A$ is meromorphic (whereas previously we considered it to be a polynomial in $h$ ) but we will see that we can adopt the theory explained in this section, to this situation too. We have

$$
P(h, z)=-a_{1} \cdot a_{2} \ldots a_{N-1} \cdot\left(h+\frac{1}{h}\right)+z^{N}+c_{1} z^{N-1}+\cdots+c_{N}
$$

Let us assume that $a_{1} \cdot a_{2} \ldots a_{N-1} \neq 0$ and pose

$$
Q(h, z) \equiv \frac{P(h, z)}{\prod_{j=1}^{N-1} a_{j}}=h+\frac{1}{h}+\frac{z^{N}+c_{1} z^{N-1}+\cdots+c_{N}}{\prod_{j=1}^{N-1} a_{j}}=h+\frac{1}{h}+d_{0} z^{N}+d_{1} z^{N-1}+\cdots+d_{N} .
$$

In $\mathbb{P}^{2}(\mathbb{C})$, the affine algebraic curve of equation $Q(h, z)=0$, is singular at infinity and to determine the genus $g$ of its normalization, we proceed as follows : note that the curve $C$ appears as a double sheeted covering of $\mathbb{P}^{1}(\mathbb{C})$ branched into $2 N$ points coinciding with the fixed points of involution $\sigma$ (3.12), that is, points where $h= \pm 1$. Using the Riemann-Hurwitz formula, we obtain

$$
g=2\left(g\left(\mathbb{P}^{1}(\mathbb{C})\right)-1\right)+1+\frac{2 N}{2}=N-1 .
$$

Consider the covering $C \longrightarrow \mathbb{P}^{1}(\mathbb{C})$ below and $\mathscr{P}, \mathscr{Q}$ located on two separate sheets. By putting $\frac{1}{z}(\infty)=\mathscr{P}+\mathscr{Q}$, we see from the equation $Q(h, z)=0$, that the divisor of $h$ is $(h)=N \mathscr{P}-N \mathscr{Q}$ and in that case, the divisor $D$ is written $D=N \mathscr{P}+N \mathscr{Q}$, which implies that $B \in H^{0}\left(D, \operatorname{Hom}(V, V(D))\right.$. The residue $\varsigma(B) \in H^{0}\left(D, \mathscr{O}_{D}(D)\right.$ satisfies the conditions of theorem 2.7 , and consequently the linear flow is given by the application (2.8). To compute the residue $\varsigma(B)$ of $B$, we will determine a set of holomorphic eigenvectors, using the van Moerbeke-Mumford method described above. Let us calculate the residue in $\mathscr{Q}$ and the result will be similarly deduced in $\mathscr{P}$. Consider a general divisor $E$ of degree $g$, of the form $E=\sum_{j=1}^{g} r_{j}$ such that : $\operatorname{dim} \mathscr{L}(E+(k-1) \mathscr{P}-k \mathscr{Q})=0$, for all $k$. We deduce from Riemann-Roch's theorem that $\operatorname{dim} \mathscr{L}(E+k \mathscr{P}-k \mathscr{Q}) \geq 1$, and therefore $\operatorname{dim} \mathscr{L}(E+k \mathscr{P}-k \mathscr{Q})=1$, for all $k$. Let

$$
\left(f_{k}\right) \in \mathscr{L}(E+k \mathscr{P}-k \mathscr{Q})=H^{0}\left(C, \mathscr{O}_{C}(E+k \mathscr{P}-k \mathscr{Q})\right), \quad 1 \leq k \leq N
$$

be a basis with $f_{N}=h$. We can choose a vector $v$ of the following form $v=\left(f_{1}, \ldots, f_{N}\right)^{\top}$, such that $v$ is an eigenvector of $A$, i.e., $A v=z v$, $(h, z) \in C$. Hence, $V=h^{-1} v$ is a holomorphic eigenvector. Without restricting generality, we take $N=3$. The system $A v=z v$, is written explicitly

$$
\begin{aligned}
b_{1} f_{1}+a_{2} f_{2}+a_{3} & =z f_{1}, \\
a_{1} f_{1}+b_{2} f_{2}+a_{2} h & =z f_{2}, \\
a_{3} h f_{1}+a_{2} f_{2}+b_{3} h & =z h .
\end{aligned}
$$

By multiplying each equation of this system by $\frac{1}{h}$, everything becomes holomorphic except the last equation, i.e., $a_{3} f_{1}=z+$ Taylor. Recall that the section of $\mathscr{O}_{D}(D)$ induced by $\lambda$ in the equation (2.7) $: B v=\dot{v}+\lambda v$, is the residue $\varsigma(B)$ of $B$. In other words,

$$
B v=\varsigma(B) v+\text { Taylor, }
$$

and therefore

$$
\left(\begin{array}{c}
\frac{a_{1} f_{2}}{h}-\frac{a_{3}}{h} \\
-\frac{a_{1} f_{1}}{h}+a_{2} \\
a_{3} f_{1}-\frac{a_{2} f_{2}}{h}
\end{array}\right)=\left(\begin{array}{c}
0 \\
0 \\
z
\end{array}\right)+\text { Taylor. }
$$

We deduce that $\varsigma(B)=\frac{z}{h}$, and $\varsigma(\dot{B})=0$. The same conclusion holds for the residue in $\mathscr{P}$. Consequently, the flow in question linearizes on the Jacobian variety of $C$. 


\section{Algebraically integrable systems}

Consider the nonlinear system of differential equations :

$$
\begin{aligned}
\frac{d z_{1}}{d t} & =f_{1}\left(t, z_{1}, \ldots, z_{n}\right), \\
& \vdots \\
\frac{d z_{n}}{d t} & =f_{n}\left(t, z_{1}, \ldots, z_{n}\right),
\end{aligned}
$$

where $f_{1}, \ldots, f_{n}$ are functions of $n+1$ complex variables $t, z_{1}, \ldots, z_{n}$ and which apply a domain of $\mathbb{C}^{n+1}$ into $\mathbb{C}$. The Cauchy problem is the search for a solution $\left(z_{1}(t), \ldots, z_{n}(t)\right)$ in a neighborhood of a point $t_{0}$, satisfying the initial conditions $: z_{1}\left(t_{0}\right)=z_{1}^{0}, \ldots, z_{n}\left(t_{0}\right)=z_{n}^{0}$. The system (4.1) can be written in vector form in $\mathbb{C}^{n}$,

$$
\frac{d z}{d t}=f(t, z(t)), \quad z=\left(z_{1}, \ldots, z_{n}\right), \quad f=\left(f_{1}, \ldots, f_{n}\right) .
$$

In this case, the Cauchy problem will be to determine the solution $z(t)$ such that $z\left(t_{0}\right)=z_{0}=\left(z_{1}^{0}, \ldots, z_{n}^{0}\right)$. When the functions $f_{1}, \ldots, f_{n}$ are holomorphic in the neighborhood of $\left(t_{0}, z_{1}^{0}, \ldots, z_{n}^{0}\right)$, then the Cauchy problem admits a holomorphic solution and only one. A question arises : can the Cauchy problem admits some non-holomorphic solution around $\left(t_{0}, z_{1}^{0}, \ldots, z_{n}^{0}\right)$ ? When $f_{1}, \ldots, f_{n}$ are holomorphic, the answer is negative. Other circumstances may arise for the Cauchy problem concerning the system of differential equations (4.1), when the holomorphic hypothesis relative to the functions $f_{1}, \ldots, f_{n}$ is no longer satisfied in the neighborhood of a point. In such a case, it can be seen that the behavior of the solutions can take on the most diverse aspects. In general, the singularities of the solutions are of two types : mobile or fixed, depending on whether or not they depend on the initial conditions. Important results have been obtained by Painlevé [31]. Suppose that the system (4.1) is written in the form

$$
\begin{aligned}
\frac{d z_{1}}{d t} & =\frac{P_{1}\left(t, z_{1}, \ldots, z_{n}\right)}{Q_{1}\left(t, z_{1}, \ldots, z_{n}\right)}, \\
& \vdots \\
\frac{d z_{n}}{d t} & =\frac{P_{n}\left(t, z_{1}, \ldots, z_{n}\right)}{Q_{n}\left(t, z_{1}, \ldots, z_{n}\right)},
\end{aligned}
$$

where

$$
\begin{gathered}
P_{k}\left(t, z_{1}, \ldots, z_{n}\right)=\sum_{0 \leq i_{1}, \ldots, i_{n} \leq p} A_{i_{1}, \ldots, i_{n}}^{(k)}(t) z_{1}^{i_{1}} \ldots z_{n}^{i_{n}}, 1 \leq k \leq n, \\
Q_{k}\left(t, z_{1}, \ldots, z_{n}\right)=\sum_{0 \leq j_{1}, \ldots, j_{n} \leq q} B_{j_{1}, \ldots, j_{n}}^{(k)}(t) z_{1}^{j_{1}} \ldots z_{n}^{j_{n}}, 1 \leq k \leq n,
\end{gathered}
$$

polynomials with several indeterminate $z_{1}, \ldots, z_{n}$ and algebraic coefficients in $t$. There are two cases: (i) the fixed singularities are constituted by four sets of points. The first set contains the singular points of the coefficients $A_{i_{1}, \ldots, i_{n}}^{(k)}(t), B_{j_{1}, \ldots, j_{n}}^{(k)}(t)$ intervening in the polynomials $P_{k}\left(t, z_{1}, \ldots, z_{n}\right)$ and $Q_{k}\left(t, z_{1}, \ldots, z_{n}\right)$. In general this set contains $t=\infty$. The second set consists of the points $\alpha_{j}$ such that : $Q_{k}\left(t, z_{1}, \ldots, z_{n}\right)=0$, which occurs if all the coefficients $B_{j_{1}, \ldots, j_{n}}^{(k)}(t)$ vanish for $t=\alpha_{j}$. The third is the set of points $\beta_{l}$ such that for some values $\left(z_{1^{\prime}}, \ldots, z_{n^{\prime}}\right)$ of $\left(z_{1}, \ldots, z_{n}\right)$, we have $P_{k}\left(\beta_{l}, z_{1^{\prime}}, \ldots, z_{n^{\prime}}\right)=Q_{k}\left(\beta_{l}, z_{1^{\prime}}, \ldots, z_{n^{\prime}}\right)=0$. Then the second members of the above system are presented in the indeterminate form $\frac{0}{0}$ at the points $\left(\beta_{l}, z_{1^{\prime}}, \ldots, z_{n^{\prime}}\right)$. Finally, the set of points $\gamma_{n}$ such that there exist $u_{1}, \ldots, u_{n}$, for which $R_{k}\left(\gamma_{n}, u_{1}, \ldots, u_{n}\right)=S_{k}\left(\gamma_{n}, u_{1}, \ldots, u_{n}\right)=0$, where $R_{k}$ and $S_{k}$ are polynomials in $u_{1}, \ldots, u_{n}$ obtained from $P_{k}$ and $Q_{k}$ by setting $z_{1}=\frac{1}{u_{1}}, \ldots, z_{n}=\frac{1}{u_{n}}$. Each of these sets contains only a finite number of elements. The system in question has a finite number of fixed singularities. (ii) the mobile singularities of solutions of this system are algebraic : poles and (or) algebraic critical points. There are no essential singular points for the solution $\left(z_{1}, \ldots, z_{n}\right)$.

We will use the method of indeterminate coefficients to find sufficient conditions for the existence and uniqueness of the meromorphic solution of the Cauchy problem concerning the system (4.1). The solution will be expressed in the form of Laurent expansions in $t$ and such a solution is formal because we obtain it by performing on various series, which we assume a priori convergent, various operations whose validity remains to be justified. The problem of convergence will therefore arise. The result will therefore be established as soon as we have verified that these series are convergent. This will be done using the majorant method [32]-[34]. Without restricting the generality, we consider the Cauchy problem relative to the normal system (4.1) where $f_{1}, \ldots, f_{n}$ do not depend explicitly on $t$, i.e.,

$$
\begin{aligned}
\frac{d z_{1}}{d t} & =f_{1}\left(z_{1}, \ldots, z_{n}\right), \\
& \vdots \\
\frac{d z_{n}}{d t} & =f_{n}\left(z_{1}, \ldots, z_{n}\right) .
\end{aligned}
$$

We suppose that $f_{1}, \ldots, f_{n}$ are rational functions in $z_{1}, \ldots, z_{n}$ and that the system (4.2) is weight-homogeneous, i.e., there exist positive integers $l_{1}, \ldots, l_{n}$ such that :

$$
f_{i}\left(\alpha^{l_{1}} z_{1}, \ldots, \alpha^{l_{n}} z_{n}\right)=\alpha^{l_{i}+1} f_{i}\left(z_{1}, \ldots, z_{n}\right), \quad 1 \leq i \leq n,
$$


for each non-zero constant $\alpha$. Note that if the determinant $\operatorname{det}\left(z_{j} \frac{\partial f_{i}}{\partial z_{j}}-\delta_{i j} f_{i}\right)_{1 \leq i, j \leq n} \not \equiv 0$, then the numbers $s_{1}, \ldots, s_{n}$ are unique. In order to facilitate the notations, we will assume (without loss of generalities) that $t_{0}=z_{0}=0$.

Theorem 4.1. Suppose that

$$
z_{i}=\frac{1}{t^{k_{i}}} \sum_{k=0}^{\infty} z_{i}^{(k)} t^{k}, \quad 1 \leq i \leq n, \quad z^{(0)} \neq 0
$$

$\left(k_{i} \in \mathbb{Z}\right.$, some $\left.k_{i}>0\right)$ is the formal solution (Laurent series), obtained by the method of undetermined coefficients of the weight-homogeneous system (4.2). Then the coefficients $z_{i}^{(0)}$ satisfy the nonlinear equation

$$
k_{i} z_{i}^{(0)}+f_{i}\left(z_{1}^{(0)}, \ldots, z_{n}^{(0)}\right)=0, \quad 1 \leq i \leq n
$$

while $z_{i}^{(1)}, z_{i}^{(2)}, \ldots$ are solution of the following system of linear equations :

$$
(L-k \mathscr{I}) z^{(k)}=\text { some polynomial in the } z^{(j)}, \quad 0 \leq j \leq k
$$

where $z^{(k)}=\left(z_{1}^{(k)}, \ldots, z_{n}^{(k)}\right)^{\top}$ and $L \equiv\left(\frac{\partial f_{i}}{\partial z_{j}}\left(z^{(0)}\right)+\delta_{i j} k_{i}\right)_{1 \leq i, j \leq n}$, is the Jacobian matrix. Moreover, the formal series (4.3) are convergent.

The coefficients $z_{i}^{(k)}$ are determined unequivocally with the adopted method of calculation which explains why the series (4.3) is the only meromorphic solution. Moreover, the result of the previous theorem applies to the following quasi-homogeneous differential equation of order $n$ :

$$
\frac{d^{n} z}{d t^{n}}=f\left(z, \frac{d z}{d t}, \ldots, \frac{d^{n-1} z}{d t^{n-1}}\right)
$$

$f$ being a rational function in $z, \frac{d z}{d t}, \ldots, \frac{d^{n-1} z}{d t^{n-1}}$ and $z(0)=z_{1}^{0}, \frac{d z}{d t}(0)=z_{2}^{0}, \ldots, \frac{d^{n-1} z}{d t^{n-1}}(0)=z_{n}^{0}$. Indeed, the differential equation above reduces to the following system :

$$
z(t)=z_{1}(t), \quad \frac{d z}{d t}(t)=z_{2}(t), \ldots, \frac{d^{n-1} z}{d t^{n-1}}(t)=z_{n}(t)
$$

We thus obtain

$$
\frac{d z_{1}}{d t}=z_{2}, \frac{d z_{2}}{d t}=z_{3}, \ldots, \frac{d z_{n-1}}{d t}=z_{n}, \frac{d z_{n}}{d t}=f\left(z_{1}, z_{2}, \ldots, z_{n}\right) .
$$

Such a system constitutes a particular case of the normal system (4.2).

Let $X_{H}$ be a Hamiltonian vector field defined by

$$
\dot{z} \equiv \frac{d z}{d t}=J \frac{\partial H}{\partial z} \equiv f(z), \quad z \in \mathbb{R}^{m}
$$

where $J=J(z)$ is a skew-symmetric matrix polynomial in $z$ of rank $2 n$, such that the Poisson bracket $\{H, F\}=\left\langle\frac{\partial H}{\partial z}, J \frac{\partial F}{\partial z}\right\rangle$ satisfies the Jacobi identity. The system (4.4) is algebraic complete integrable (in abbreviated form : a.c.i.) when $J$ has polynomial entries and when the following conditions hold :

i) The system is completely integrable with polynomial invariants $H_{1}, \ldots, H_{n+k}$. It means that besides the $k$ invariants $H_{1}, \ldots, H_{k}$ (Casimir functions), i.e., such that $J \frac{\partial H_{i}}{\partial z}(z)=0,1 \leq i \leq k$, the system admits $n=\frac{m-k}{2}$ invariants $H_{k+1}=H, \ldots, H_{k+n}$ in involution, i.e., such that $\left\{H_{i}, H_{j}\right\}=0$. These give rise to $n$ commuting vector fields. For generic $c_{i}$, the invariant manifolds (level surfaces)

$$
\bigcap_{i=1}^{n+k}\left\{z \in \mathbb{R}^{m}: H_{i}=c_{i}\right\},
$$

are compact, connected and therefore real tori according to the Arnold-Liouville theorem [12].

ii) The invariant manifolds (level surfaces) thought of as lying in $\mathbb{C}^{m}$,

$$
\bigcap_{i=1}^{n+k}\left\{z \in \mathbb{C}^{m}: H_{i}=c_{i}\right\},
$$

are related, for generic $c_{i}$, to Abelian varieties $T^{n}=\mathbb{C}^{n} /$ Lattice (complex algebraic tori) as follows :

$$
\bigcap_{i=1}^{n+k}\left\{z \in \mathbb{C}^{m}: H_{i}=c_{i}\right\}=T^{n} \backslash D,
$$

where $D$ is a divisor (codimension one subvarieties) in $T^{n}$. The coordinates $z_{i}$ are meromorphic on $T^{n}$ and $D$ is the minimal divisor on $T^{n}$ where the variables $z_{i}$ blow up. The flows (4.4) run with complex time are straight-line motions on $T^{n}$. 
As the reader has surely noted, we have insisted in the above definition that invariants must be polynomials. But it must be understood that the existence of a sufficient number of polynomial invariants does not necessarily imply the algebraic complete integrability of the system in question. To convince ourselves of this, it is enough to consider the following Hamiltonian system whose Hamiltonian is

$$
H\left((x, y)=\frac{x^{2}}{2}+P_{n}(y)\right.
$$

where $P_{n}(y)$ is a polynomial in $y$ of degree $n$. We show that such a system is algebraically completely integrable if and only if $n=3$ or 4 , and the explicit resolution of the system is done using elliptic functions. So a natural question arises : given a completely integrable system with polynomial invariants, what makes it algebraically completely integrable? Mumford gives in his book [21] a definition of the algebraic complete integrability including also non compact and explains (although it has nothing to do with the system above) this extra feature as follows : the vector fields $X_{H_{1}}, \ldots, X_{H_{n}}$ define on the real torus $M_{c}=\bigcap_{i=1}^{n+k}\left\{H_{i}=c_{i}\right\} \subset \mathbb{R}^{2 n}$ an addition law

$$
\oplus: M_{c} \times M_{c} \longrightarrow M_{c},(x, y) \longmapsto x \oplus y=g_{t+s}(p), \quad p \in M_{c},
$$

with $x=g_{t}(p), y=g_{s}(p), g_{t}(p)=g_{t_{1}}^{X_{1}} \ldots g_{t_{n}}^{X_{n}}(p)$, where $g_{t_{i}}^{X_{i}}(p)$ denote the flow of $X_{H_{i}}$. From the polynomial nature of the vector fields X, this addition law will always be real analytic. The algebraic complete integrability of the system in question means that this law of addition is rational. In other words, we have $(x \oplus y)_{j}=R_{j}\left(x_{i}, y_{i}, c\right)$, where $R_{j}\left(x_{i}, y_{i}, c\right)$ is a rational function of the coordinates $x_{i}, y_{i}$ for all $i=1,2, \ldots, n$. Putting $x=p, y=g_{t}^{X_{i}}(p)$, in the above formula, we notice that on the real torus $M_{c}$, the flows $g_{t}^{X_{i}}(p)$ depend rationally on the initial condition $p$. Moreover, a Weierstrass theorem on the functions admitting a law of addition, affirms that the coordinates $x_{i}$ restricted to the real torus :

$$
\mathbb{R}^{n} / \text { lattice } \longrightarrow M_{c}, \quad\left(t_{1}, \ldots, t_{n}\right) \longmapsto z_{i}\left(t_{1}, \ldots, t_{n}\right),
$$

are Abelian functions. Geometrically, this means that the real torus $M_{c} \approx \mathbb{R}^{n} /$ lattice is the affine part of an algebraic complex torus (Abelian variety) $T^{n} \simeq \mathbb{C}^{n} /$ lattice and the real functions $z_{i}\left(t_{1}, \ldots, t_{n}\right),\left(t_{i} \in \mathbb{R}\right)$, are the restrictions to this real torus of meromorphic functions $z_{i}\left(t_{1}, \ldots, t_{n}\right),\left(t_{i} \in \mathbb{C}\right)$ of $n$ complex variables, with $2 n$ real periods (of which $n$ real periods and $n$ imaginary periods). It must be said that Mumford's explanation of the algebraic complete integrability of a completely integrable Hamiltonian system with polynomial invariants, is of purely theoretical interest. Indeed, how do you recognise from the differential equations that, on a given level manifold, the commuting vector fields define a rational addition law ? Painlevé [31] provides the following provocative example, among many others not necessarily in the context of Hamiltonian mechanics. Consider on $\mathbb{C}^{2}$ the two polynomial commuting vector fields :

$$
\begin{array}{llll}
X_{1}: & \dot{x}=x, & \dot{y}=x y, \\
X_{2}: & \dot{x}=0, & \dot{y}=y .
\end{array}
$$

The flow

$$
g_{t}^{X_{1}}\left(x_{0}, y_{0}\right)=\left(x_{0} e^{t}, y_{0}^{x_{0}\left(e^{t}-1\right)}\right)
$$

doest not depend rationally on the initial condition $\left(x_{0}, y_{0}\right)$. Therefore, simply looking at the face of the equations does not answer the question of whether the problem is algebraically completely integrable. The only method was to solve the problem explicitly in terms of Abelian integrals.

Now if the system (4.4) is algebraically completely integrable, it means that the variables $z_{i}$ restricted to a generic complex invariant manifold of the flows, are meromorphic functions on a complex torus $\mathbb{C}^{n} /$ lattice; in fact these are Abelian functions. By compactness, these functions must blow up along a divisor (a codimension one subvariety) $D \subset \mathbb{C}^{n}$ /lattice. Expanding the solutions of the system (4.4) near this divisor and allowing the constants of the motion to vary, one gets meromorphic solutions depending on $\operatorname{dim} D+\sharp H_{i}=m-1$ parameters, because $\operatorname{dim} D=n-1$ and $\sharp H_{i}=n+k$ is the number of constants of the motion. The fact that algebraic complete integrable systems possess $(m-1)$-dimensional families of Laurent solutions, was implicitly used by Kowalewski [35] in her classification of integrable rigid body motions. The following necessary condition was developed and used by Adler-van Moerbeke [36] :

Theorem 4.2. Suppose that the Hamiltonian system (4.4) is algebraically completely integrable with Abelian functions $z_{i}$ and for generic $c$, the invariant tori related to this system do not contain elliptic curves. Then this system must admit enough meromorphic Laurent expansion solutions in $t \in \mathbb{C}$ such that : each $z_{i}$ blows up at least once and Laurent expansion of $z_{i}$, depend on $m-1$, free parameters. In addition, the system in question has families of Laurent solutions depending on $m-2, m-3, \ldots, m-n$, parameters and the coefficients of each of these solutions are rational functions on affine algebraic varieties of dimensions $m-1, m-2, m-3, \ldots, m-n$.

The question is whether this criterion is sufficient and how it can be used to detect algebraically completely integrable systems. The idea of the direct proof given by Adler-van Moerbeke[37, 38] is closely related to the geometric spirit of the real Arnold-Liouville theorem [12]. Namely, a compact complex $n$-dimensional variety on which there exist $n$ holomorphic commuting vector fields which are independent at every point is analytically isomorphic to a $n$-dimensional complex torus $\mathbb{C}^{n} /$ Lattice and the complex flows generated by the vector fields are straight lines on this complex torus. Now a complex affine algebraic variety is never compact, unless it is 0-dimensional. So the main problem will be to complete the affine variety $M_{c}=\bigcap_{i=1}^{n+k}\left\{z \in \mathbb{C}^{m}, H_{i}=c_{i}\right\}$, into a non-singular compact complex algebraic variety $\bar{M}_{c}=M_{c} \cup D$ in such a way that the vector fields extend holomorphically along $D$ and remain independent there. If this is possible, $\bar{M}_{c}$ is an Abelian variety (an algebraic complex torus) and the coordinates $z_{i}$ restricted to $M_{c}$ are Abelian functions. To compactifize $M_{c}$ into an algebraic complex torus, a naive guess would be to take the natural compactification

$$
\bar{M}_{c}=\bigcap_{i=1}^{n+k}\left\{Z \in \mathbb{P}^{m}(\mathbb{C}), H_{i}(Z)=c_{i} Z_{0}^{\operatorname{deg} H_{i}}\right\},
$$


of $M_{c}$ by projectivizing the equations. Indeed, this can never work for a general reason: an Abelian variety $\widetilde{M}_{c}$ of dimension bigger or equal than two is never a complete intersection, that is it can never be described in some projective space $\mathbb{P}^{n}(\mathbb{C})$ by $n$-dim $\widetilde{M}_{c}$ global polynomial homogeneous equations. In other words, if $M_{c}$ is to be the affine part of an Abelian variety, $\bar{M}_{c}$ must have a singularity somewhere along the locus at infinity, i.e., along all or part of the hyperplane section $\left\{Z_{0}=0\right\}$ at infinity. The trajectories of the vector fields (4.4) hit every point of the singular locus at infinity and ignore the smooth locus at infinity. In fact, the existence of meromorphic solutions to the differential equations (4.4) depending on some free parameters can be used to manufacture the tori, without ever going through the delicate procedure of blowing up and down. Information about the tori can then be gathered from the divisor. A partial converse to theorem 4.2, can be formulated as follows [36] :

Theorem 4.3. We assume that condition $i)$ in the above definition of the algebraic complete integrability is satisfied. In addition, suppose that the system (4.4) with $k+n$ polynomial invariants have a coherent tree of Laurent solutions, i.e., it possesses families of Laurent solutions in $t$, depending on $n-1, n-2, \ldots, m-n$, free parameters. Then, this system is algebraic complete integrable and moreover, there are no other Laurent solutions of $m-1$ dimension than those provided by the coherent set.

The study of the algebraic complete integrability of Hamiltonian systems, includes several passages to prove rigorously. Here we mention the main passages. We saw that if the flow is algebraically completely integrable, the differential equations (4.4) must admits Laurent series solutions

$$
z_{i}(t)=\frac{1}{t^{k_{i}}}\left(z_{i}^{(0)}+z_{i}^{(1)}+\cdots\right), \quad k_{i} \in \mathbb{Z}, \quad i=1,2, \ldots
$$

where $z_{i}^{(0)}, z_{i}^{(1)}, \ldots$ are rational functions depending on $m-1$, free parameters. We must have $k_{i}=l_{i}$ and coefficients in the series must satisfy at the $0^{\text {th }}$ step nonlinear equations,

$$
f_{i}\left(z_{1}^{(0)}, \ldots, z_{m}^{(0)}\right)+g_{i} z_{i}^{(0)}=0,1 \leq i \leq m
$$

and at the $k^{\text {th }}$ step, linear systems of equations :

$$
(\mathscr{M}-k I) z^{(k)}=\left\{\begin{aligned}
0 & \text { for } k=1 \\
\text { polynomials in } & z^{(1)}, \ldots, z^{(k-1)} \text { for } k>1
\end{aligned}\right.
$$

where $\mathscr{M}=\frac{\partial f}{\partial z}+\left.g I\right|_{z=z^{(0)}}$ is the Jacobian matrix of the equations (4.5). If $m-1$, free parameters are to appear in the Laurent series, they must either come from the nonlinear equations (4.5) or from the eigenvalue problem (4.6), i.e., $\mathscr{M}$ must have at least $m-1$, integer eigenvalues. These are much less conditions than expected, because of the fact that the homogeneity $k$ of the constant $H$ must be an eigenvalue of $L$. The formal series solutions are convergent as a consequence of the majorant method. By substituting these series solutions into the constants of motion $H_{i}(z), 1 \leq i \leq n+k$, one eliminates some parameters linearly, leading to an algebraic relation between the remaining parameters, which is nothing but the equation of the divisor $D$ along which the $z_{i}$ blow up; if the differential equations admit $l$ families of Laurent meromorphic solutions of the form above, it means that $D$ is formed by $l$ algebraic curves. More precisely, you have to prove that the set

$$
D \equiv\left\{z_{i}(t), 1 \leq i \leq m, \text { Laurent solutions such that }: H_{j}\left(z_{i}(t)\right)=c_{j}+\text { Taylor part }\right\}
$$

defines one or several $n-1$ dimensional algebraic varieties ("Painlevé" divisor) having the property that $\bigcap_{i=1}^{n+k}\left\{z \in \mathbb{C}^{m}: H_{i}=c_{i}\right\} \cup D$, is a smooth compact, connected variety with $n$ commuting vector fields independent at every point, i.e., a complex algebraic torus $\mathbb{C}^{n} /$ lattice Note that the system of coordinates $z_{1}, \ldots, z_{m}$ can be enlarged to a new set $z_{0}=1, z_{1}, \ldots, z_{N}$ having the property that for fixed but arbitrary $0 \leq j \leq N$, we have

$$
\overbrace{\left(\frac{z_{i}}{z_{j}}\right)}^{i}=\frac{\dot{z}_{i} z_{j}-z_{i} \dot{z}_{j}}{z_{j}^{2}}=\sum_{k, l} a_{k, l}\left(\frac{z_{k}}{z_{j}}\right)\left(\frac{z_{l}}{z_{j}}\right)
$$

i.e., the ratios $\frac{z_{i}}{z_{j}}$ form a closed system of coordinates under differentiation. Indeed, consider a point $p \in D$, a chart $U_{j}$ around $p$ on the torus and a function $z_{j}$ in $L(D)$ having a pole of maximal order at $p$. Then the vector $\left(\frac{1}{z_{j}}, \frac{z_{1}}{z_{j}}, \ldots, \frac{z_{N}}{z_{j}}\right)$ provides a good system of coordinates in $U_{j}$. Then taking the derivative with regard to one of the flows $\overbrace{\left(\frac{z_{i}}{z_{j}}\right)}^{i}$ are finite on $U_{j}$ as well. Therefore, since $z_{j}^{2}$ has a double pole along $D$, the numerator must also have a double pole (at worst), i.e., $\dot{z}_{i} z_{j}-z_{i} \dot{z}_{j} \in L(2 D)$. Hence, when the divisor $D$ is projectively normal, i.e., whenever $L(k D)=L(D)^{\otimes k}$ which means that the space $L(k D)$ is generated by homogeneous polynomials of degree $k$ in some basis elements of $L(D)$. At the bad points, the concept of projective normality play an important role: this enables one to show that $\frac{z_{i}}{z_{j}}$ is a bona fide Taylor series starting from every point in a neighborhood of the point in question. Therefore, the flows $J \frac{\partial H_{k+i}}{\partial z}, \ldots, J \frac{\partial H_{k+n}}{\partial z}$ are straight line motions on this torus (for concrete applications, see for example [32, 36, 39, 40, 41, 42, 43]). Let's point out that having computed the space of functions $\mathscr{L}(D)$ with simple poles at worst along with the expansions, it is often important to compute the space of functions $\mathscr{L}(k D)$ of functions having $k$-fold poles at worst along with the expansions. These functions play a crucial role in the study of the procedure for embedding the invariant tori into projective space. As mentioned previously, the idea of the Adler-van Moerbeke's proof $[37,38]$ consists of using arguments similar to those used in the proof of the real Arnold-Liouville theorem [12], and we can call this result the Liouville-Arnold-Adler-van Moerbeke theorem: 
Theorem 4.4. Let $\widetilde{M}$ be an $n$-dimensional complex compact manifold with $n$ independent meromorphic functions. Assume that : (i) For some divisor $D$, there exist $n$ non-vanishing holomorphic vector fields $X_{1}, \ldots, X_{n}$ on the affine variety $\widetilde{M} \backslash D=M$ which commute and are independent at every point.

(ii) One vector field, say $X_{k}$ (where $1 \leq k \leq n$ ), extends holomorphically on $\widetilde{M}$ and having the property that, for all $p \in D$,

$$
\left\{g_{t_{k}}^{X_{k}}(p): 0<|t|<\varepsilon, t \in \mathbb{C}\right\} \subset M,
$$

where $g_{t_{k}}^{X_{k}}$ denote the flow of $X_{k}$. This condition means that the orbits of $X_{k}$ through $D$ go immediately into the affine part $M$ and in particular, the vector field $X_{k}$ does not vanish on any point of $D$.

Then, $\widetilde{M}$ is an Abelian variety and the vector fields $X_{1}, \ldots, X_{n}$ extend holomorphically and remain independent on $\widetilde{M}$.

1) As an example, consider the Kac-van Moerbeke periodic lattice [44] given by the following system :

$$
\dot{x}_{j}=x_{j}\left(x_{j-1}-x_{j+1}\right), \quad j=1, \ldots, 5
$$

where $\left(x_{1}, \ldots, x_{5}\right) \in \mathbb{C}^{5}$ and $x_{j}=x_{j+5}$. This vector field forms a Hamiltonian system for the Poisson structure

$$
\left\{x_{j}, x_{k}\right\}=x_{j} x_{k}\left(\delta_{j, k+1}-\delta_{j+1, k}\right), \quad 1 \leq j, k \leq 5
$$

and admits three independent first integrals

$$
\begin{aligned}
& H_{1}=x_{1} x_{3}+x_{2} x_{4}+x_{3} x_{5}+x_{4} x_{1}+x_{5} x_{2}, \\
& H_{2}=x_{1}+x_{2}+x_{3}+x_{4}+x_{5}, \\
& H_{3}=x_{1} x_{2} x_{3} x_{4} x_{5} .
\end{aligned}
$$

Note that $H_{1}$ and $H_{2}$ are involution while $H_{3}$ is a Casimir, and the system in question is therefore integrable. The affine manifold

$$
\bigcap_{j=1}^{3}\left\{x=\left(x_{1}, x_{2}, x_{3}, x_{4}, x_{5}\right) \in \mathbb{C}^{5}: H_{j}(x)=c_{j}\right\}, \quad\left(c_{1}, c_{2}, c_{3}\right) \in \mathbb{C}^{3}, \quad c_{3} \neq 0
$$

is isomorphic to $\operatorname{Jac}(C) \backslash D$ where $C$ is a curve of genus 2 given by the equation.

$$
w^{2}=\left(z^{3}-c_{1} z^{2}+c_{2} z\right)^{2}-4 z
$$

and $D$ consists of five copies of $C$ in the Jacobian variety $\operatorname{Jac}(C)$. The flows generated by $H_{1}$ et $H_{2}$ are linearized on $\operatorname{Jac}(C)$ and the system is algebraically completely integrable. The reader interested in the study of this system via various methods can find further information with more detail in [39] as well as in [45].

2) The problem we are going to study now is the generalized periodic Toda systems. We consider $l+1$ vectors $e_{0}, \ldots, e_{l}$ in the Euclidean vector space $\left(\mathbb{R}^{l+1},\langle. \mid\rangle.\right), l \geq 1$, linearly dependent and such that they are $l$ to $l$ linearly independent (i.e, for all $j$, the vectors $e_{0}, \ldots, \widehat{e}_{j}, \ldots, e_{l}$ are linearly independent). Suppose that the non-zero reals $\xi_{0}, \ldots, \xi_{l}$ satisfying $\sum_{j=0}^{l} \xi_{j} e_{j}=0$ are non-zero sum; that is, $\sum_{j=0}^{l} \xi_{j} \neq 0$. Let $\Omega=\left(a_{i j}\right)_{0 \leq i, j \leq l}$ be the matrix where

$$
a_{i j}=2 \frac{\left\langle e_{i} \mid e_{j}\right\rangle}{\left\langle e_{j} \mid e_{j}\right\rangle}, \quad 0 \leq i, j \leq l
$$

We consider the vector field $X_{\Omega}$ on $\mathbb{C}^{2(l+1)}$, defined by

$$
\dot{x}=x . y \quad \dot{y}=A x, \quad\left(x, y \in \mathbb{C}^{l+1}\right),
$$

where $x . y=\left(x_{0} y_{0}, \ldots, x_{l} y_{l}\right)$. It has been shown [32] that if $X_{\Omega}$ is an integrable vector field of an irreducibly algebraically completely integrable system, then $\Omega$ is the Cartan matrix of a twisted affine Lie algebra. Specific detailed results concerning this problem can be found on the technical paper [39] and also in [32, 46] and references therein, about link between Abelian varieties, Dynkin diagrams, singularities and Toda lattice. The periodic $l+1$ particle Toda lattices are associated to extended Dynkin diagrams. They are completely integrable and have as many polynomial invariants as points in the Dynkin diagram. The affine variety defined by the intersections of the constants of the motion is completed into an Abelian variety by the addition of a specific divisor $D$. The latter consists of $l+1$ irreducible components $D_{j}$ each associated with a root $\alpha_{j}$ of the extended Dynkin diagram $\Delta$. The intersection of $k$ components $D_{j_{1}}, \ldots, D_{j_{k}}$ satisfies the following relation : the intersection multiplicity of the intersection of $k$ components of the divisor equals $\frac{\operatorname{order}(W)}{\operatorname{det}(\Omega)}$ where $W$ and $A$ are the Weyl group and the Cartan matrix going with the sub-Dynkin diagram $\alpha_{j_{1}}, \ldots, \alpha_{j_{k}}$ associated with the $k$ components. The intersection of all the divisors except one is a discrete set of points whose number is explicitly determined, but on the other hand the intersection of all the divisors are empty. The set-theoretical number of points is given (in terms of the Dynkin diagram) by

$$
\text { Number of }\left(\bigcap_{\beta \neq \alpha} D_{\beta}\right)=\frac{p_{\alpha}}{p_{0}}\left(\frac{\operatorname{order}\left(\text { Weyl group of the Dynkin diagram } \Delta \backslash \alpha_{0}\right)}{\operatorname{order}(\text { Weyl group of the Dynkin diagram } \Delta \backslash \alpha)}\right),
$$

where the integers $p_{\alpha}$, are given by the null vector of the Cartan matrix (going with the extended Dynkin diagram $\Delta$ ). The singularities of the divisor are canonically associated to semi-simple Dynkin diagrams and those of each component occur only at the intersections with other 
components and their multiplicities at the intersection with other divisors are expressed in terms of how a corresponding root is located in the sub-Dynkin diagram determined by this root and those of the members of the above divisor intersection. We have

$$
\operatorname{sing}\left(D_{k}\right) \subseteq D_{k} \cap \sum_{\substack{0 \leq j \leq l \\ j \neq k}} D_{j}, \quad k=0, \ldots, l
$$

and this inclusion is valid for the singular locus $\operatorname{sing}\left(D_{k}\right)$ of $D_{k}$. The multiplicity of the singularity of a particular component $D_{k}$, at its intersection with $m$ other divisors is entirely specified by the way the corresponding root $\alpha_{k}$ are located in the sub-Dynkin diagram $\alpha_{k}, \alpha_{j_{1}}, \ldots, \alpha_{j_{m}}$. (See [39], for proof of these results as well as other information).

There are many examples of Hamiltonian systems, called algebraic completely integrable in the generalized sense, for which all movable singularities of the general solution have only a finite number of branches and the complex invariant manifolds are coverings of Abelian varieties. These systems of differential equations possess solutions which are Laurent expansions containing $n$-th root terms of type $\sqrt[n]{t}(t$ being complex time) and whose coefficients depend rationally on certain algebraic parameters. In other words, for these systems just replace in the above definition of the complete algebraic integrability of Hamiltoian systems, the condition $i i)$ by by this one,

iii) the invariant manifolds $\bigcap_{i=1}^{n+k}\left\{z \in \mathbb{C}^{m}: H_{i}=c_{i}\right\}$ are related to an $l$-fold cover $\widetilde{T}^{n}$ of the torus $T^{n}$ ramified along a divisor $D$ in $T^{n}$ as follows

$$
\bigcap_{i=1}^{n+k}\left\{z \in \mathbb{C}^{m}: H_{i}=c_{i}\right\}=\widetilde{T}^{n} \backslash D
$$

Let $H_{m}$ be a family of Hamiltonians $[47,48]$ :

$$
H_{m}(x, y)=\frac{1}{2}\left(p_{x}^{2}+p_{y}^{2}\right)+\alpha_{m} V_{m}(x, y), \quad m=1,2, \ldots
$$

where

$$
V_{m}(x, y)=\sum_{k=0}^{[m / 2]} \frac{(m-k) ! 2^{m-2 k}}{k !(2 k-m) !} x^{2 k} y^{m-2 k}, \quad m=1,2, \ldots
$$

It is easy to verify that the associated Hamiltonian systems have a second first integral :

$$
F_{m}(x, y)=p_{x}\left(x p_{y}-y p_{x}\right)+\alpha_{m} x^{2} V_{m-1}(x, y), \quad m=1,2, \ldots
$$

and they are Liouville integrable. The study of the systems corresponding to the cases $m \geq 3$ is not obvious contrary to the cases $m=1$ and $m=2$ whose study is immediate. For $m=3$, the study is reduced to that of the Hénon-Heiles system [49]:

$$
\begin{aligned}
& \dot{y}_{1}=x_{1}, \\
& \dot{y}_{2}=x_{2}, \\
& \dot{x}_{1}=-\varepsilon y_{1}-2 y_{1} y_{2}, \\
& \dot{x}_{2}=-y_{1}^{2}-16 \varepsilon y_{2}-16 y_{2}^{2},
\end{aligned}
$$

corresponding to a generalized Hénon-Heiles Hamiltonian

$$
H=\frac{1}{2}\left(x_{1}^{2}+x_{2}^{2}\right)+\frac{\varepsilon}{2}\left(y_{1}^{2}+16 y_{2}^{2}\right)+y_{1}^{2} y_{2}+\frac{16}{3} y_{2}^{3},
$$

where $y_{1}, y_{2}, x_{1}, x_{2}$ are canonical coordinates and momenta respectively and $\varepsilon$ a constant parameter. The associated Hamiltonian system has the following second constant of motion :

$$
F=3 x_{1}^{4}+6 \varepsilon x_{1}^{2} y_{1}^{2}+12 x_{1}^{2} y_{1}^{2} y_{2}-4 x_{1} x_{2} y_{1}^{3}-4 \varepsilon y_{1}^{4} y_{2}-4 y_{1}^{4} y_{2}^{2}+3 \varepsilon^{2} y_{1}^{4}-\frac{2}{3} y_{1}^{6} .
$$

The functions $H$ and $F$ commute $:\{H, F\}=\sum_{k=1}^{2}\left(\frac{\partial H}{\partial x_{k}} \frac{\partial F}{\partial y_{k}}-\frac{\partial H}{\partial y_{k}} \frac{\partial F}{\partial x_{k}}\right)=0$. The system (4.7) admits Laurent solutions in $\sqrt{t}$, depending on three free parameters : $\alpha, \beta, \gamma$ and they are explicitly given as follows

$$
\begin{aligned}
y_{1} & =\frac{\alpha}{\sqrt{t}}+\beta t \sqrt{t}-\frac{\alpha}{18} t^{2} \sqrt{t}+\frac{\alpha \varepsilon^{2}}{10} t^{3} \sqrt{t}-\frac{\alpha^{2} \beta}{18} t^{4} \sqrt{t}+\cdots, \\
y_{2} & =-\frac{3}{8 t^{2}}-\frac{\varepsilon}{2}+\frac{\alpha^{2}}{12} t-\frac{2 \varepsilon^{2}}{5} t^{2}+\frac{\alpha \beta}{3} t^{3}-\gamma t^{4}+\cdots, \\
x_{1} & =-\frac{1}{2} \frac{\alpha}{t \sqrt{t}}+\frac{3}{2} \beta \sqrt{t}-\frac{5}{36} \alpha t \sqrt{t}+\frac{7}{20} \alpha \varepsilon^{2} t^{2} \sqrt{t}-\frac{1}{4} \alpha^{2} \beta t^{3} \sqrt{t}+\cdots, \\
x_{2} & =\frac{3}{4 t^{3}}+\frac{1}{12} \alpha^{2}-\frac{4}{5} \varepsilon^{2} t+\alpha \beta t^{2}-4 \gamma t^{3}+\cdots
\end{aligned}
$$


As previously mentioned, the convergence of these series results from the majorant method. By replacing these series in the equations $H=a$, $F=b$, one eliminates one parameter linearly, leading to an algebraic relation between the two remaining parameters, which is nothing but the equation of an algebraic curve $D$ along which the $\left(y_{1}(t), y_{2}(t), x_{1}(t), x_{2}(t)\right)$ blow up. To be more precise, we have

$$
\begin{aligned}
& H=\frac{1}{9} \alpha^{2}-\frac{21}{4} \gamma+\frac{13}{288} \alpha^{4}+\frac{4}{3} \varepsilon^{3}=a, \\
& F=-144 \alpha \beta^{3}+\frac{294}{5} \alpha^{3} \beta \varepsilon^{2}+\frac{8}{9} \alpha^{6}-33 \gamma \alpha^{4}=b,
\end{aligned}
$$

which implies that

$$
144 \alpha \beta^{3}-\frac{294 \varepsilon^{2}}{5} \alpha^{3} \beta+\frac{143}{504} \alpha^{8}-\frac{4}{21} \alpha^{6}+\frac{44}{21}\left(4 \varepsilon^{3}-3 a\right) \alpha^{4}+b=0
$$

Let

$$
A=\bigcap_{k=1}^{2}\left\{\left(y_{1}, y_{2}, x_{1}, x_{2}\right) \in \mathbb{C}^{4}: H\left(y_{1}, y_{2}, x_{1}, x_{2}\right)=a, F\left(y_{1}, y_{2}, x_{1}, x_{2}\right)=b\right\},
$$

be the smooth affine surface defined by putting the two invariants $H$ and $F$ equal to generic constants $a$ and $b$. The Laurent expansions above where $\left(y_{1}(t), y_{2}(t), x_{1}(t), x_{2}(t)\right)$ blow up contain square root terms of the type $\sqrt{t}$ and admit three free parameters and in addition these solutions restricted to the surface $A$ are parameterized by the curve $D$. We will see that (4.7) is in fact a generalized algebraic completely integrable system but is part of a new system that is algebraically completely integrable. This latter is a system of five nonlinear differential equations with five unknowns having three first integrals, two of which are cubic and one is quartic. By inspection of the expansions (4.8), we look for polynomials in $\left(y_{1}, y_{2}, x_{1}, x_{2}\right)$ without fractional exponents, which suggests considering the following change of variables :

$$
z_{1}=y_{1}^{2}, \quad z_{2}=y_{2}, \quad z_{3}=x_{2}, \quad z_{4}=y_{1} x_{1}, \quad z_{5}=3 x_{1}^{2}+2 y_{1}^{2} y_{2} .
$$

Note that this change of variables determines a morphism on the affine variety $A$ (4.9). Using the two first integrals $H, F$ and differential equations (4.7), we obtain the following system :

$$
\begin{aligned}
& \dot{z}_{1}=2 z_{4}, \\
& \dot{z}_{2}=z_{3}, \\
& \dot{z}_{3}=-z_{1}-16 \varepsilon z_{2}-16 z_{2}^{2}, \\
& \dot{z}_{4}=-\varepsilon z_{1}-\frac{8}{3} z_{1} z_{2}+\frac{1}{3} z_{5}, \\
& \dot{z}_{5}=2 z_{1} z_{3}-8 z_{2} z_{4}-6 \varepsilon z_{4},
\end{aligned}
$$

having two cubic and one quartic invariants (constants of motion),

$$
\begin{aligned}
G_{1} & =\frac{1}{2} \varepsilon z_{1}+\frac{1}{6} z_{5}+8 \varepsilon z_{2}^{2}+\frac{1}{2} z_{3}^{2}+\frac{2}{3} z_{1} z_{2}+\frac{16}{3} z_{2}^{3} \\
G_{2} & =9 \varepsilon^{2} z_{1}^{2}+z_{5}^{2}+6 \varepsilon z_{1} z_{5}-2 z_{1}^{3}-24 \varepsilon z_{1}^{2} z_{2}-12 z_{1} z_{3} z_{4}+24 z_{2} z_{4}^{2}-16 z_{1}^{2} z_{2}^{2} \\
G_{3} & =z_{1} z_{5}-3 z_{4}^{2}-2 z_{1}^{2} z_{2} .
\end{aligned}
$$

This new system is a completely integrable Hamiltonian system where $G_{1}$ is the Hamiltonian whose structure is determined by the bracket

$$
\{H, F\}=\left\langle\frac{\partial H}{\partial z}, J \frac{\partial F}{\partial z}\right\rangle
$$

the anti-symmetric matrix $J$ defines a Poisson structure for which the corresponding Poisson bracket satisfies the Jacobi identity. The two first integrals $G_{1}$ and $G_{2}$ are in involution while the latter $G_{3}$ is trivial (i.e., a Casimir function). For generic values of constants $c_{1}, c_{2}$ and $c_{3}$, the invariant variety

$$
B=\bigcap_{k=1}^{3}\left\{\left(z_{1}, z_{2}, z_{3}, z_{4}, z_{5}\right) \in \mathbb{C}^{5}: G_{k}\left(z_{1}, z_{2}, z_{3}, z_{4}, z_{5}\right)=c_{k}\right\},
$$

is a smooth affine surface. The differential equations (4.11) admit Laurent series expansions restricted to the surface $B$ (4.12); these solutions can be read off from (4.8) and the change of variable (4.10) and depend on four free parameters. We have shown that the change of variables (4.10) transforms the system (4.7) into an algebraic completely integrable system (4.11) of five differential equations in five unknowns and parallel to that, the affine variety $A$ (4.9) is transformed into the affine part $B$ (4.12) of an Abelian variety $\widetilde{B}$. The Hamiltonian system (4.7) is a generalized algebraic complete integrable system, the invariant surface $A$ (4.9) can be completed as a cyclic double cover $\bar{A}$ of an Abelian surface $\widetilde{B}$ and in addition, $\bar{A}$ is smooth except at the point lying over the singularity of type $A_{3}$ whose resolution $\widetilde{A}$ of $\bar{A}$ is a surface of general type. This explains (among other) why the asymptotic solutions to the differential equations (4.7) contain fractional powers. All this is summarized as follows [50] :

Theorem 4.5. The system (4.7) admits Laurent solutions with fractional powers depending on three free parameters and is algebraic complete integrable in the generalized sense. In addition, this system is part of a new system of differential equations (4.11) in five unknowns having two cubic and one quartic invariants (constants of motion). This last system possesses Laurent expansions (but without fractional powers) depending on four free parameters and it is algebraically completely integrable. 
The case $m=4$ corresponds to Ramani-Dorizzi-Grammaticos (RDG) potential [47, 48], whose corresponding system is given by

$$
\ddot{q}_{1}-q_{1}\left(q_{1}^{2}+3 q_{2}^{2}\right)=0, \quad \ddot{q}_{2}-q_{2}\left(3 q_{1}^{2}+8 q_{2}^{2}\right)=0 .
$$

These equations can be written in the form of an integrable Hamiltonian system whose Hamiltonian is given by

$$
H_{1}=\frac{1}{2}\left(p_{1}^{2}+p_{2}^{2}\right)-\frac{3}{2} q_{1}^{2} q_{2}^{2}-\frac{1}{4} q_{1}^{4}-2 q_{2}^{4}
$$

The second first integral being

$$
H_{2}=p_{1}^{4}-6 q_{1}^{2} q_{2}^{2} p_{1}^{2}+q_{1}^{4} q_{2}^{4}-q_{1}^{4} p_{1}^{2}+q_{1}^{6} q_{2}^{2}+4 q_{1}^{3} q_{2} p_{1} p_{2}-q_{1}^{4} p_{2}^{2}+\frac{1}{4} q_{1}^{8} .
$$

The first integrals $H_{1}$ and $H_{2}$ are obviously in involution. For generic $\left(b_{1}, b_{2}\right) \in \mathbb{C}^{2}$, the affine variety $B$ defined by

$$
B=\bigcap_{k=1}^{2}\left\{z \in \mathbb{C}^{4}: H_{k}(z)=b_{k}\right\}
$$

is a smooth surface. The solutions of the differential equations (4.13) in the form of Laurent's series depend on three free parameters $u, v, w$. and are written

$$
\begin{aligned}
& q_{1}=\frac{1}{\sqrt{t}}\left(u-\frac{1}{4} u^{3} t+v t^{2}-\frac{5}{128} u^{7} t^{3}+\frac{1}{8} u\left(\frac{3}{4} u^{3} v-\frac{7}{256} u^{8}+3 \kappa w\right) t^{4}+\cdots\right) \\
& q_{2}=\frac{1}{t}\left(\frac{1}{2} \kappa-\frac{1}{4} \kappa u^{2} t+\frac{1}{8} \kappa u^{4} t^{2}+\frac{1}{4} \kappa u\left(\frac{1}{32} u^{5}-3 v\right) t^{3}+w t^{4}+\cdots\right) \\
& p_{1}=\frac{1}{2 t \sqrt{t}}\left(-u-\frac{1}{4} u^{3} t+3 v t^{2}-\frac{25}{128} t^{3} u^{7}+\frac{7}{8} u\left(\frac{3}{4} u^{3} v-\frac{7}{256} u^{8}+3 \kappa w\right) t^{4}+\cdots\right) \\
& p_{2}=\frac{1}{t^{2}}\left(-\frac{1}{2} \kappa+\frac{1}{8} \kappa u^{4} t^{2}+\frac{1}{2} \kappa u\left(\frac{1}{32} u^{5}-3 v\right) t^{3}+3 w t^{4}+\cdots\right)
\end{aligned}
$$

where $\kappa= \pm 1$. The convergence of these series derives from the the majorant method. Note that these solutions contain square root terms of type $\sqrt{t}$, and we will see that these terms can be removed by introducing the variables $z_{1}, z_{2}, z_{3}, z_{4}, z_{5}(4.17)$ which restores the Painlevé property (that is, the only singularities are poles) of the system in question. Substituting (4.15) in the invariants $H_{1}=b_{1}$ and $H_{2}=b_{2}$, after eliminating the parameter $w$, we obtain the following equation (of a curve of genus 16 denoted $\Gamma$ ) connecting the parameters $u$ and $v$ :

$$
a_{1} u v^{3}+a_{2} u^{6} v^{2}-a_{3} u^{11} v+a_{4} b_{1} u^{3} v-a_{5} u^{16}-a_{6} b_{1} u^{8}+b_{2}+a_{7}=0 .
$$

where $a_{1}=\frac{65}{4}, a_{2}=\frac{93}{64}, a_{3}=\frac{29487}{8192}, a_{4}=\frac{78336}{8192}, a_{5}=\frac{10299}{65536}, a_{6}=\frac{123}{256}, a_{7}=\frac{1536298731}{52}$. Consider on the variety $B(4.14)$, the following morphism

$$
\psi: \mathscr{B} \longrightarrow \mathbb{C}^{5}, \quad\left(q_{1}, q_{2}, p_{1}, p_{2}\right) \longmapsto\left(z_{1}, z_{2}, z_{3}, z_{4}, z_{5}\right)
$$

where

$$
z_{1}=q_{1}^{2}, \quad z_{2}=q_{2}, \quad z_{3}=p_{2}, \quad z_{4}=q_{1} p_{1}, \quad z_{5}=p_{1}^{2}-q_{1}^{2} q_{2}^{2}
$$

These variables are easily obtained by simple inspection of the series (4.15). By using the variables (4.17) and differential equations (4.13), one obtains

$$
\begin{aligned}
& \dot{z}_{1}=2 z_{4}, \\
& \dot{z}_{2}=z_{3} \\
& \dot{z}_{3}=z_{2}\left(3 z_{1}+8 z_{2}^{2}\right) \\
& \dot{z}_{4}=z_{1}^{2}+4 z_{1} z_{2}^{2}+z_{5}, \\
& \dot{z}_{5}=2 z_{1} z_{4}+4 z_{2}^{2} z_{4}-2 z_{1} z_{2} z_{3} .
\end{aligned}
$$

This new system on $\mathbb{C}^{5}$ admits the following three first integrals

$$
\begin{aligned}
& F_{1}=\frac{1}{2} z_{5}-z_{1} z_{2}^{2}+\frac{1}{2} z_{3}^{2}-\frac{1}{4} z_{1}^{2}-2 z_{2}^{4} \\
& F_{2}=z_{5}^{2}-z_{1}^{2} z_{5}+4 z_{1} z_{2} z_{3} z_{4}-z_{1}^{2} z_{3}^{2}+\frac{1}{4} z_{1}^{4}-4 z_{2}^{2} z_{4}^{2} \\
& F_{3}=z_{1} z_{5}+z_{1}^{2} z_{2}^{2}-z_{4}^{2} .
\end{aligned}
$$

The first integrals $F_{1}$ and $F_{2}$ are in involution, while $F_{3}$ is trivial (Casimir function). The invariant variety $A$ defined by

$$
A=\bigcap_{k=1}^{3}\left\{\left(z_{1}, z_{2}, z_{3}, z_{4}, z_{5}\right) \in \mathbb{C}^{5}: F_{k}\left(z_{1}, z_{2}, z_{3}, z_{4}, z_{5}\right)=c_{k}\right\},
$$


is a smooth affine surface for generic values of $\left(c_{1}, c_{2}, c_{3}\right) \in \mathbb{C}^{3}$. The system (4.18) is completely integrable and possesses Laurent series solutions which depend on four free parameters $\alpha, \beta, \gamma$ et $\theta$ :

$$
\begin{aligned}
z_{1} & =\frac{1}{t} \alpha-\frac{1}{2} \alpha^{2}+\beta t-\frac{1}{16} \alpha\left(\alpha^{3}+4 \beta\right) t^{2}+\gamma t^{3}+\cdots \\
z_{2} & =\frac{1}{2 t} \kappa-\frac{1}{4} \kappa \alpha+\frac{1}{8} \kappa \alpha^{2} t-\frac{1}{32} \kappa\left(-\alpha^{3}+12 \beta\right) t^{2}+\theta t^{3}+\cdots \\
z_{3} & =-\frac{1}{2 t^{2}} \kappa+\frac{1}{8} \kappa \alpha^{2}-\frac{1}{16} \kappa\left(-\alpha^{3}+12 \beta\right) t+3 \theta t^{2}+\cdots \\
z_{4} & =-\frac{1}{2 t^{2}} \alpha+\frac{1}{2} \beta-\frac{1}{16} \alpha\left(\alpha^{3}+4 \beta\right) t+\frac{3}{2} \gamma t^{2}+\cdots \\
z_{5} & =\frac{1}{2 t^{2}} \alpha^{2}-\frac{1}{4 t}\left(\alpha^{3}+4 \beta\right)+\frac{1}{4} \alpha\left(\alpha^{3}+2 \beta\right)-\left(\alpha^{2} \beta-2 \gamma+4 \kappa \theta \alpha\right) t+\cdots,
\end{aligned}
$$

where $\kappa= \pm 1$. The convergence of these series is guaranteed by the majorant method. By replacing these series in the equations $F_{1}=c_{1}$, $F_{2}=c_{2}, F_{3}=c_{3}$ one eliminates two parameters $\gamma$ and $\theta$ linearly, leading to an algebraic relation between the two remaining parameters, which is the equation of an algebraic curve $C$ of genus 7 ,

$$
64 \beta^{3}-16 \alpha^{3} \beta^{2}-4\left(\alpha^{6}-32 \alpha^{2} c_{1}-16 c_{3}\right) \beta+\alpha\left(32 c_{2}-32 \alpha^{4} c_{1}+\alpha^{8}-16 \alpha^{2} c_{3}\right)=0 .
$$

The Laurent solutions restricted to the surface $A$ (4.20) are thus parameterized by two copies $C_{-1}$ and $C_{1}$ of the same Riemann surface $C$ (4.22) and we embed these curves in a hyperplane of $\mathbb{P}^{15}(\mathbb{C})$ using the sixteen functions :

$$
\begin{aligned}
& 1, \quad z_{1}, \quad z_{2}, \quad 2 z_{5}-z_{1}^{2}, \quad z_{3}+2 \kappa z_{2}^{2}, \quad z_{4}+\kappa z_{1} z_{2}, \quad W\left(f_{1}, f_{2}\right), \quad f_{1}\left(f_{1}+2 \kappa f_{4}\right), \quad f_{2}\left(f_{1}+2 \kappa f_{4}\right), \quad z_{4}\left(f_{3}+2 \kappa f_{6}\right), \\
& z_{5}\left(f_{3}+2 \kappa f_{6}\right), \quad f_{5}\left(f_{1}+2 \kappa f_{4}\right), \quad f_{1} f_{2}\left(f_{3}+2 \kappa f_{6}\right), \quad f_{4} f_{5}+W\left(f_{1}, f_{4}\right), \quad W\left(f_{1}, f_{3}\right)+2 \kappa W\left(f_{1}, f_{6}\right), \quad f_{3}-2 z_{5}+4 f_{4}^{2},
\end{aligned}
$$

where $W\left(s_{j}, s_{k}\right) \equiv \dot{s}_{j} s_{k}-s_{j} \dot{s}_{k}$ is the Wronskian. The curves $C_{1}$ and $C_{-1}$ have double points in common where they are tangent to each other and which are a singularity of type $A_{3}$ of $C_{1}+C_{-1}$. The Hamiltonian system (4.13) is algebraic complete integrable in the generalized sense and the invariant surface $B$ (4.14) is completed as a cyclic double cover $\bar{B}$ of the Abelian surface $\widetilde{A}$, ramified along the divisor $C_{1}+C_{-1}$. In addition, $\bar{B}$ is smooth except at the singularity above and the resolution $\widetilde{B}$ of $\bar{B}$ is a surface of general type. Let $G$ be a cyclic group of two elements $\{-1,1\}$ on $V_{\varepsilon}^{j}=U_{\varepsilon}^{j} \times\{\tau \in \mathbb{C}: 0<|\tau|<\delta\}$, where $\tau=\sqrt{t}$ and $U_{\varepsilon}^{j}$ is an affine chart of $\Gamma_{\varepsilon}$ for which the Laurent expansions (4.21) are well defined. Since the action of $G$ is defined by $(-1) \circ(u, v, \tau)=(-u,-v,-\tau)$ and is without fixed points in $V_{\varepsilon}^{j}$, then the quotient $V_{\varepsilon}^{j} / G$ identifies itself with the image of the smooth map $h_{\varepsilon}^{j}: V_{\varepsilon}^{j} \longrightarrow B$ defined by the Laurent series (4.21). We have

$$
(-1,1) \cdot(u, v, \tau)=(-u,-v, \tau), \quad(1,-1) \cdot(u, v, \tau)=(u, v,-\tau),
$$

which means that $G \times G$ acts separately on each coordinate and so,, identifying $V_{\varepsilon}^{j} / G^{2}$ with the image of $\psi \circ h_{\varepsilon}^{j}$ in $A$. Note that, except for a finite number of points, $B_{\varepsilon}^{j}=V_{\varepsilon}^{j} / G$ is smooth and the coherence of the $B_{\varepsilon}^{j}$ follows from the coherence of $V_{\varepsilon}^{j}$ and the action of $G$. After gluing various varieties $B_{\varepsilon}^{j} \backslash$ \{some points on $B$, we obtain a smooth complex manifold $\widehat{B}$ which is a double cover of the Abelian variety $\widetilde{A}$ ramified along $C_{1}+C_{-1}$, and therefore can be completed to an algebraic cyclic cover of $\widetilde{A}$. We would like to know information on the points that are missing. For this, we must examine the image of $\Gamma \times\{0\}$ in $\cup B_{\mathcal{\varepsilon}}^{j}$. The quotient $\Gamma \times\{0\} / G$ is birationally equivalent to the curve $\Upsilon$ defined by the equation :

$$
a_{1} y^{3}+a_{2} x^{3} y^{2}-a_{3} x^{6} y+a_{4} b_{1} x^{2} y-\left(a_{5} x^{8}+a_{6} b_{1} x^{4}-b_{2}-a_{7}\right) x=0
$$

and its genus is 7 , where $a_{1}, \ldots, a_{7}$, have been defined above and $y=u v, x=u^{2}$. The curve $\Upsilon$ is birationally equivalent to $C$ and the only points of $\Upsilon$ fixed under $(u, v) \longmapsto(-u,-v)$ are the points at $\infty$. These correspond to the (double) ramification points of the map $\Gamma \times\{0\} \longrightarrow \Upsilon:(u, v) \longmapsto(x, y)$, and coincide with the points at $\infty$ of the curve $C$. The variety $\widehat{B}$ constructed above is birationally equivalent to the compactification $\bar{B}$ of $B$ and $\bar{B}$ is a cyclic double cover of the Abelian surface $\widetilde{A}$. The system (4.13) is algebraic complete integrable in the generalized sense and $\bar{B}$ is smooth except at the point lying over the singularity (of type $A_{3}$ ) of $C_{1}+C_{-1}$. The resolution $\widetilde{B}$ of singularities of $\bar{B}$, is a surface of general type with invariants : Euler characteristic of $\widetilde{B}=1$ and geometric genus of $\widetilde{B}=2$. In conclusion, we have [51],

Theorem 4.6. The Hamiltonian system (4.13) is algebraic complete integrable in the generalized sense and possess Laurent expansions depending on three free parameters : $u, v, w$, and containing square root terms of type $\sqrt{t}$. These Laurent solutions restricted to the affine manifold $B$ (4.14) are parameterized by two copies $\Gamma_{1}$ and $\Gamma_{-1}$ of an algebraic curve $\Gamma$ (4.16) of genus 16 . This system is part of a new algebraically completely integrable system (4.18) in five unknowns and having three quartics invariants (4.19). The complex invariant

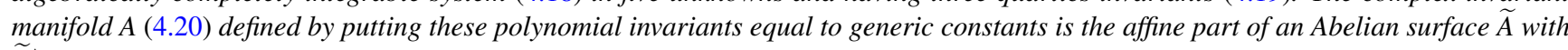
$\widetilde{A} \backslash A=C_{1}+C_{-1}$, where the divisor $C_{1}+C_{-1}$ is very ample and consists of two components $C_{1}$ and $C_{-1}$ of a genus 7 curve $C$ (4.22). In addition, the invariant manifold $B$ is completed into a cyclic double cover $\bar{B}$ of the Abelian surface $\widetilde{A}$, ramified along the divisor $C_{1}+C_{-1}$ in such a way that the vector fields extend holomophically alond this divisor and remain independent there. Moreover, $\bar{B}$ is smooth except at the point lying over the singularity (of type $A_{3}$ ) of $C_{1}+C_{-1}$ and the resolution $\widetilde{B}$ of $\bar{B}$ is a surface of general type with invariants : Euler characteristic of $\widetilde{B}=1$ and geometric genus of $\widetilde{B}=2$.

\section{Conclusion}

At the end of this paper, it is worth to mention some similar problems as well as recent results. Abelian varieties, very heavily studied by algebraic geometers, enjoy certain algebraic properties which can then be translated into differential equations and their Laurent solutions. Among the results presented in this paper, there is an explicit calculation of invariants for Hamiltonian systems which cut out an open set in 
an Abelian variety and various algebraic curves related to these systems are given explicitly. The integrable systems presented here are interesting problems, particular to experts of Abelian varieties who may want to see explicit examples of correspondence for varieties defined by different algebraic curves. The methods used are primarily analytical but heavily inspired by algebraic geometrical methods. The concept of algebraic complete integrability is quite effective in small dimensions and has the advantage to lead to global results, unlike the existing criteria for real analytic integrability, which, at this stage are perturbation results. In fact, the overwhelming majority of dynamical systems, Hamiltonian or not, are non-integrable and possess regimes of chaotic behavior in phase space. The methods used are primarily analytical but heavily inspired by algebraic geometrical methods. Abelian varieties and cyclic coverings of Abelian varieties, very heavily studied by algebraic geometers, enjoy certain algebraic properties which can be translated into differential equations and their Laurent solutions.

In recent years, other important results have been obtained following studies on the KP and KdV hierarchies. The use of tau functions related to infinite dimensional Grassmannians, Fay identities, vertex operators and the Hirota's bilinear formalism led to obtaining remarkable properties concerning these algebras of infinite order differential operators as for example the existence of an infinite family of first integrals functionally independent and in involution. The elaboration of powerful methods and the discovery of their common algebraic structures led to important developments concerning the study of nonlinear problems. The functions $\tau(t)$ are specific functions of time, constructed from sections of a determinant bundle on an infinite-dimensional Grassmannian manifold. These functions generalize the Riemann theta functions and they are solutions of the KP hierarchy, i.e, solutions of an infinite series of nonlinear partial differential equations connecting infinity of functions of infinity variables. The functions $\tau(t)$ can be Schur polynomials, falling within Fredholm's group representation theory or determinants. Recently, a new type of tau function has appeared, within the framework of quantum gauge theory with gauge group $S U(N)$ when $N$ is large. This led to the so-called matrix models (quantum gravity) for counting triangulations on certain surfaces (topology). The underlying models have remained relatively intractable except in two space-time dimensions; although being physically toy models, their structure is still very rich. The first tau function was introduced by Sato, Miwa, and Jimbo in relation to the theory of isomonodromic deformations. It has been defined as a correlation function of certain quantum fields associated with the poles of a Fuchsian system on the Riemann sphere. These functions give information on the topology of moduli spaces of Riemann surfaces and are closely related to the theory of representations of Virasoro algebras and W-algebras. The $\tau(t)$ functions play an important role in a large number of branches of mathematics and theoretical physics, such as integrable systems, string theories, quantum-gauge theories, isomonodromic deformations, matrix models (quantum gravity), the associated matrix integrals which have power series expansions (perturbative series) and whose terms count the triangulations on surfaces (Feynman graphs), the module problems and in many other domains. Many problems related to algebraic geometry, combinatorics, probabilities and quantum gauge theory,..., have been solved explicitly by methods inspired by techniques from the study of integrable systems. In particular, the study of random matrices, a domain that establishes links with several problems, for example with combinatorics, probabilities, number theory, models of growth and random tailings and questions of communication technology. The functions $\tau(t)$ are the source of inspiration for many mathematicians and physicists in search of new algebraic structures appearing in mathematics and physics. The vertex operators give a good device to the investigation of the matrix models and the spectrum of the stochastic matrices. An account of these results will appear elsewhere.

\section{References}

[1] H. Poincaré, Leçons de Mécanique Céleste, 3 tomes. Gauthier-Villars, 1905-1910, réédité par Jacques Gabay, Paris 2003.

[2] D. J. Korteweg, G. de Vries, On the change of form of long waves advancing in a rectangular canal and on a new type of long stationary waves, Phil. Mag., 39 (1895), 422-443.

[3] C. G. J. Jacobi, Vorlesungen über Dynamik, Königsberg lectures of 1842-1843, reprinted by Chelsea Publishing Co., New York, 1969.

[4] P. A. Griffiths, Linearizing flows and a cohomological interpretation of Lax equations, Amer. J. Math., 107 (1985), $1445-1483$.

[5] M. Adler, On a trace functional for formal pseudo differential operators and the symplectic structure of the Korteweg-de Vries type equations, Invent. Math., 50(3) (1979), 219-248.

[6] B. Kostant, The solution to a generalized Toda lattice and representation theory, Adv. Math., 34(3) (1979), 195-338.

[7] W. Symes, Systems of Toda type, inverse spectral problems and representation theory, Invent. Math., 59 (1980), 13-53.

[8] M. Adler, P. van Moerbeke, The AKS theorem, A.C.I. systems and random matrix theory, J. Phys. A, 51(42) (2018).

[9] M. Adler, P. van Moerbeke, Completely integrable systems, Euclidean Lie algebras, and curves, Adv. in Math., 38 (1980), 267-379.

[10] M. Adler, P. van Moerbeke, Linearization of Hamiltonian systems, Jacobi varieties and representation theory, Adv. in Math., 38 (1980), $318-379$.

[11] P. van Moerbeke, D. Mumford, The spectrum of difference operators and algebraic curves, Acta Math., 143 (1979), 93-154.

[12] V. I. Arnold, Mathematical Methods in Classical Mechanics, Springer-Verlag, Berlin-Heidelberg- New York, 1978, 2nd edn., Graduate Texts in Mathematics, Vol. 60, Springer-Verlag, New York, 1989.

[13] C. Neumann, De problemate quodam mechanics, quod ad primam integralium ultraellipticorum classem revocatur, J. Reine Angew. Math., 56 (1859), 46-63.

[14] H. Knörrer, Integrable Hamiltonsche Systeme und algebraische Geometrie, Jahresber. Deutsch. Math.- Verein., 88 (2) (1986), $82-103$.

[15] H. Knörrer, Geodesics on the ellipsoïd, Invent. Math., 59 (1980), 119-143.

[16] H. Knörrer, Geodesics on quadrics and a mechanical problem of C. Neumann, J. Reine Angew. Math., 334 (1982), 69-78.

[17] P. Deift F. Lund, E. Trubowitz, Nonlinear wave equations and constrained harmonic motion, Comm. Math. Phys., 74 (1980), $141-188$.

[18] J. L. Lagrange, Mécanique analytique, Oeuvres de Lagrange, Serret J.A. [Darboux G.] eds., t. 11, Gauthier-Villars, Paris, 1888.

[19] T. Ratiu, P. van Moerbeke, The Lagrange rigid body motion, Ann. Inst. Fourier, 32(1) (1982), 211-234.

[20] T. Ratiu, Euler-Poisson equations on Lie algebras and the n-dimensional heavy rigid body, Amer. J. Math., 104 (1982), 409-448.

[21] D. Mumford, Tata lectures on theta I, II, Progress in Math., Birkhaüser, Boston, 1983.

[22] E. Arbarello, M. Cornalba, P. Griffiths, J. Harris, Geometry of algebraic curves, I. Grundlehren der mathematischen Wissenschaften, 267, Springer-Verlag, New York, 1985

[23] S. V. Manakov, Remarks on the integrals of the Euler equations of the n-dimensional Heavy Top, Fund. Anal. Appl., 10 (4) (1976), 93-94.

[24] P. van Moerbeke, The spectrum of Jacobi matrices, Invent. Math., 37 (1976), 45-81.

[25] H. Flaschka, The Toda lattice I, Existence of integrals, Phys. Rev., B 3, 9 (1974), 1924-1925.

[26] J. Moser, Various Aspects of Integrable Hamiltonian Systems, In: Guckenheimer J., Moser J., and Newhouse S. E., Dynamical Systems, O.I.M.E. Lectures, Bressanone, Italy, June 1978, Progr. Math. 8, Birkhäuser, Boston, 233-289, 1980.

[27] J. Moser, Geometry of quadrics and spectral theory, Lecture delivred at the symposium in honour of S.S. Chern, Berkeley, 1979. Springer, Berlin, Heidelberg, New-York, 1980.

[28] Y. Kato, On the spectral density of periodic Jacobi matrices, Proceedings of RIMS Symposium on Non-Linear Integrable Systems-Classical Theory and Quantum Theory, Kyoto Japan, 1981, 153-181, World Science Publishing Co., 1983.

[29] Y. Kato, Mixed periodic Jacobi continued fractions, Nagoya Math. J., 104 (1986), 129-148.

[30] M. Toda, Wave propagation in anharmonic lattices, J. Phys. Soc. of Japan, 23 (1967), 501-506.

[31] P. Painlevé, Oeuvres, tomes 1,2,3. Édition du C.N.R.S., 1975.

[32] M. Adler, P. van Moerbeke, P. Vanhaecke, Algebraic integrability, Painlevé geometry and Lie algebras, A series of modern surveys in mathematics, 47, Springer-Verlag, 2004.

[33] J.-P. Françoise, Integrability of quasi-homogeneous vector fields, Unpublished preprint. 
[34] E. Hille, Ordinary Differential Equations in the Complex Domain, Wiley-Interscience, New-York, 1976.

[35] S. Kowalewski, Sur le problème de la rotation d'un corps solide autour d'un point fixe, Acta Math., 12 (1889), 177-232.

[36] M. Adler, P. van Moerbeke, The complex geometry of the Kowalewski-Painlevé analysis, Invent. Math., 97 (1989), 3-51.

[37] M. Adler, P. van Moerbeke, Algebraic completely integrable systems: a systematic approach, I, II, III, Séminaire de Mathématique, Rapport No 110, p.1-145, SC/MAPA - Institut de mathématique pure et appliquée, UCL, 1985.

[38] A. Lesfari, Algebraic integrability: the Adler-van Moerbeke approach, Regul. Chaotic Dyn., 16(3-4) (2011), $187-209$.

[39] M. Adler, P. van Moerbeke, The Toda lattice, Dynkin diagrams, singularities and abelian varieties, Invent. Math., 103(2) (1991), 223-278.

[40] L. Haine, Geodesic flow on SO(4) and Abelian surfaces, Math. Ann., 263 (1983), 435-472.

[41] A. Lesfari, Abelian surfaces and Kowalewski's top, Ann. Scient. École Norm. Sup., Paris, $4^{e}$ série, 21 (1988), $193-223$.

[42] A. Lesfari, Integrable systems and complex geometry, Lobachevskii J. Math., 30(4) (2009), 292-326.

[43] P. Vanhaecke, Integrable systems in the realm of algebraic geometry, Lecture Notes in Math., 1638, Springer-Verlag, Berlin, 2001.

[44] M. Kac, P. van Moerbeke, On an explicitly soluble system of nonlinear differential equations related to certain Toda lattices, Adv. in Math., 16 (1975), $160-169$.

[45] G. Teschil, Jacobi Operators and Completely Integrable Nonlinear Lattices, Mathematical Surveys and Monographs, 72, 2000.

[46] P. A. Griffiths, J. Harris, Principles of Algebraic Geometry, Wiley-Interscience, New-York, 1978.

[47] A. Ramani, B. Dorozzi, B. Grammaticos, B. Painlevé conjecture revisited, Phys. Rev. Lett., 49 (1982), 1539-1541.

[48] B. Grammaticos, B. Dorozzi, A. Ramani, Integrability of Hamiltonians with third and fourth-degree polynomial potentials, J. Math. Phys., 24 (1983), 2289-2295.

[49] M. Hénon, C. Heiles, The applicability of the third integral of motion; some numerical experiments, Astron. J., 69 (1964), 73-79.

[50] A. Lesfari, The Hénon-Heiles system as part of an integrable system, J. Adv. Res. Dyn. Control Syst., 6(3) (2014), 24-31.

[51] A. Lesfari, Abelian varieties, surfaces of general type and integrable systems, Beiträge Algebra Geom., 48(1) (2007), 95-114. 